\title{
Moduli and (un)attractor black hole thermodynamics
}

\author{
Dumitru Astefanesei • Kevin Goldstein • \\ Swapna Mahapatra
}

Received: 6 July 2007 / Accepted: 15 January 2008 / Published online: 6 February 2008

(C) The Author(s) 2008

\begin{abstract}
We investigate four-dimensional spherically symmetric black hole solutions in gravity theories with massless, neutral scalars non-minimally coupled to gauge fields. In the non-extremal case, we explicitly show that, under the variation of the moduli, the scalar charges appear in the first law of black hole thermodynamics. In the extremal limit, the near horizon geometry is $A d S_{2} \times S^{2}$ and the entropy does not depend on the values of moduli at infinity. We discuss the attractor behaviour by using Sen's entropy function formalism as well as the effective potential approach and their relation with the results previously obtained through special geometry method. We also argue that the attractor mechanism is at the basis of the matching between the microscopic and macroscopic entropies for the extremal non-BPS Kaluza-Klein black hole.
\end{abstract}

Keywords Black holes · Attractors · Entropy function · String theory

\section{Introduction}

One of the most successful applications of string theory, as a theory of quantum gravity, has been the study of black holes and their entropy. String theory provides

\footnotetext{
D. Astefanesei

Harish-Chandra Research Institute, Allahabad 211 019, India

e-mail: dastef@mri.ernet.in

K. Goldstein ( $\varangle)$

Tata Institute of Fundamental Research, Homi Bhabha Road, Mumbai 400 005, India e-mail: k.goldstein@uu.nl; kevin@theory.tifr.res.in

S. Mahapatra

Physics Department, Utkal University, Bhubaneswar 751 004, India

e-mail: swapna@iopb.res.in
} 
a microscopic description of a special class of black holes [34,48]. Essential to the embedding of charged black holes in string theory is the notion of compactification on some internal compact manifold. A fundamental element of these constructions is that all of the stringy constituents are wrapped around some non-trivial cycles of the internal space so that the final configuration appears as point-like in the lower dimensional space. A sufficiently heavy compactified wrapped object will effectively give rise to a lower dimensional space containing a horizon, or in other words, a black hole. Roughly speaking, the microscopic picture of black holes in string theory is based on the (tiny) internal manifold where the extended objects are trapped. The geometry of internal manifold is parametrised by certain moduli. These moduli will appear as fields in the lower dimensional effective field theory.

It is well-known that the expectation value of the dilaton controls the string coupling constant $g_{s}=e^{\langle\phi\rangle}$. This tells us that the strength of the interaction is determined dynamically via the vacuum expectation value (vev) of a scalar field in the string spectrum. In fact, even the constants which appear upon compactification are vevs of certain massless scalar fields (referred to as moduli fields) and are determined dynamically by the choice of the vacuum (i.e., the choice of a consistent string background).

In this paper, we consider static four-dimensional charged black hole solutions in gravity theories with $U(1)$ gauge fields and neutral massless scalars. ${ }^{1}$ The general Lagrangian we consider includes the bosonic part of $\mathcal{N}=1$ or 2 supergravities for particular values of the couplings. We refer to the scalar fields as moduli even though they do not necessarily characterize the geometry of an internal space because they still determine the $U(1)$ couplings. The moduli have a non-trivial radial dependence and hence the properties of these black holes depend on the values $\left(\phi_{\infty}\right)$ of moduli at spatial infinity. ${ }^{2}$ Since the moduli are non-minimally coupled to gauge fields and the scalar charges are non-zero at spatial infinity, one expects a modification of the first law of black hole thermodynamics. That is, the first law of black hole thermodynamics should be supplemented by a new term containing the variation of the moduli [22]:

$$
d M=T d S+\psi^{A} d Q_{A}-\Sigma_{i} d \phi_{\infty}^{i}
$$

where $\Sigma_{i}$ are the scalar charges and $\psi^{A}$ is the potential conjugate to the $U(1)$ charges $Q_{A}$. Interestingly enough, the scalar charge is not protected by a gauge symmetry and so it is not a conserved charge. Therefore, this form of the first law should be taken with caution: in string theory the scalar fields (moduli) are interpreted as local coupling constants and so a variation of the moduli values at infinity is equivalent to changing the background. ${ }^{3}$

Unlike the non-extremal case where the near horizon geometry (and the entropy) depends on the values of the moduli at infinity, in the extremal case, the near horizon geometry is universal and is determined by only the charge parameters. Consequently,

\footnotetext{
1 While massless scalars are unnatural in a generic non-supersymmetric theory they are at least technically natural with $\mathcal{N}=1$ supersymmetry. For this reason, our results are best understood in the context of non-supersymmetric solutions of theories with $\mathcal{N} \geq 1$.

$2 \phi_{\infty}$ label different ground states (vacua) of the theory.

3 As mentioned in [22], one could imagine, for example, a cosmological scenario in which $\phi_{\infty}$ does vary.
} 
the entropy is also independent of the asymptotic values of the moduli. The scalars vary radially, but they are "attracted" to fixed values at the horizon, depending only on the charge parameters. ${ }^{4}$

The attractor mechanism was discovered in the context of four-dimensional $\mathcal{N}=2$ supergravity [19,21,47], then extended to other supergravities [20]—including actions with higher derivative corrections [33] (see, e.g., [37] for a nice review on this subject). It is now well understood that supersymmetry does not really play a fundamental role in the attractor phenomenon-the attractormechanism relies only on the form of the near horizon geometry [44]. For spherically symmetric black holes, the near horizon geometry is $A d S_{2} \times S^{2}$, which continues to ensure the attractor behaviour even after including $\alpha^{\prime}$ corrections [1,7,42-45]. In fact, it has been suggested that, the "long throat" of $A d S_{2}$ is at the basis of attractor mechanism [44,2,29].

The paper is organised as follows: in Sect. 2, we describe the general set-up involving the action, equations of motion, and the effective potential. In Sect. 3, we study the spherically symmetric non-extremal solutions, focusing on some of the exact solutions. We explicitly discuss the appearance of scalar charge in the context of first law of black hole thermodynamics. In Sect. 4, we discuss the attractor mechanism in the context of the extremal limit of our black hole solutions. We discuss the equivalence of the effective potential approach [17,25] and the entropy function [44] formalism in the near horizon limit for a specific effective potential. We show that, for the non-extremal black hole solutions whose near horizon geometry is not $A d S_{2} \times S^{2}$, the effective potential is not generically minimised and hence there is no attractor behaviour. We generalize the results of [25] to non-extremal black holes and discuss how the relationship between the effective potential and entropy is modified. We also discuss the role of attractor in the case of near-extremal black holes with large charges in string theory. Using a perturbation analysis of non-extremal Reissner-Nordstrom black holes with respect to variations of the asymptotic moduli, we explicitly find that, to first order in perturbation theory, the near horizon geometry depends on the moduli. Finally, we discuss the conditions for the attractor phenomenon in special geometry language $[17,29]$ as well as the relation with the other methods. In Sect. 5 we discuss the role of attractor mechanism in understanding the entropy of non-BPS extremal black holes. For some examples of non-supersymmetric extremal black holes in $\mathcal{N}=2$ four-dimensional supergravity [30,15] an agreement has been found between the Bekenstein-Hawking entropy and the microscopic entropy computed in string theory. It is tempting to conjecture that the deeper reason for this matching is the attractor mechanism. For $A d_{2} \times S^{2}$ geometries, the equations of motion are equivalent to extremising the entropy function which fixes the moduli. The horizon is an attractor and so the near horizon geometry is universal, determined just by charges. One of the moduli is the dilaton that controls the Newton constant and so by moving from strong coupling to weak coupling asymptotically, the entropy determined by the near horizon geometry, does not change. 5

\footnotetext{
${ }^{4}$ A similar behaviour was obtained for the rotating black holes [2]. However, in this case the values of the scalars at the horizon have also an angular dependence.

5 The authors of [11] also suggest that the attractor mechanism is at the root of this matching and carefully discuss numerous examples.
} 


\section{General set-up}

We will focus on a four-dimensional theory of gravity coupled to a set of massless scalars and vector fields, whose general bosonic action has the form ${ }^{6}$

$$
\begin{aligned}
I\left[G_{\mu \nu}, \phi^{i}, A_{\mu}^{I}\right]= & -\frac{1}{k^{2}} \int_{M} d^{4} x \sqrt{-G}\left[R-2 g_{i j}(\phi) \partial_{\mu} \phi^{i} \partial^{\mu} \phi^{j}-f_{A B}(\phi) F_{\mu \nu}^{A} F^{B \mu \nu}\right. \\
& \left.-\tilde{f}_{A B}(\phi) F_{\mu \nu}^{A} F_{\rho \sigma}^{B} \epsilon^{\mu \nu \rho \sigma}\right]+\frac{2}{k^{2}} \int_{\partial M} d^{3} x \sqrt{-h} \Theta
\end{aligned}
$$

where $F_{\mu \nu}^{A}$, with $A=(0, \ldots N)$, are the $U(1)$ gauge fields, $\phi^{i}$, with $(i=1, \ldots, n)$, are the scalar fields, $\epsilon^{\mu \nu \rho \sigma}$ is the completely antisymmetric tensor, and $k^{2}=16 \pi G_{N}$. The last term is the boundary Gibbons-Hawking term; $h_{a b}$ and $\Theta$ are the induced metric and the trace of the extrinsic curvature of the boundary geometry, respectively. The moduli determine the gauge coupling constants and $g_{i j}(\phi)$ is the metric on the moduli space. We use Gaussian units so that factors of $4 \pi$ in the gauge fields can be avoided and the Newton's constant $G_{N}$ is set to 1 . The above action resembles that of the ungauged supergravity theories.

The equations of motion for the metric, moduli, and the gauge fields are given by

$$
\begin{gathered}
R_{\mu \nu}-2 g_{i j} \partial_{\mu} \phi^{i} \partial_{\nu} \phi^{j}=f_{A B}\left(2 F_{\mu \lambda}^{A} F_{\nu}^{B}{ }_{\nu}^{\lambda}-\frac{1}{2} G_{\mu \nu} F_{\alpha \lambda}^{A} F^{B \alpha \lambda}\right) \\
\frac{1}{\sqrt{-G}} \partial_{\mu}\left(\sqrt{-G} g_{i j} \partial^{\mu} \phi^{j}\right)=\frac{1}{4} \frac{\partial f_{A B}}{\partial \phi^{i}} F_{\mu \nu}^{A} F^{B \mu \nu}+\frac{1}{8} \frac{\partial \tilde{f}_{A B}}{\partial \phi^{i}} F_{\mu \nu}^{A} F_{\rho \sigma}^{B} \epsilon^{\mu \nu \rho \sigma}, \\
\partial_{\mu}\left[\sqrt{-G}\left(f_{A B} F^{B \mu \nu}+\frac{1}{2} \tilde{f}_{A B} F_{\rho \sigma}^{B} \epsilon^{\mu \nu \rho \sigma}\right)\right]=0
\end{gathered}
$$

where we have varied the moduli and the gauge fields independently. The Bianchi identities for the gauge fields are $F^{A}{ }_{[\mu \nu ; \lambda]}=0$.

We consider the following spherically symmetric ansatz for the metric

$$
d s^{2}=-a(r)^{2} d t^{2}+a(r)^{-2} d r^{2}+b(r)^{2} d \Omega^{2} .
$$

The Bianchi identity and equation of motion for the gauge fields can be solved by a field strength of the form [25]

$$
F^{A}=f^{A B}\left(Q_{B}-\tilde{f}_{B C} P^{C}\right) \frac{1}{b^{2}} d t \wedge d r+P^{A} \sin \theta d \theta \wedge d \phi,
$$

where $P^{A}, Q_{A}$ are constants which determine the magnetic and electric charges carried by the gauge field $F^{A}$ and $f^{A B}$ is the inverse of $f_{A B}$.

\footnotetext{
6 We also consider "axionic" type couplings characterised by $\tilde{f}_{A B}$. In the so-called axion-dilaton-gravity model, the coupling is a pseudo-scalar such that the action is parity-invariant.
} 
As discussed in [25], given our ansatze the equations of motion can be written as

$$
\begin{gathered}
\left(a^{2} b^{2}\right)^{\prime \prime}=2, \\
\frac{b^{\prime \prime}}{b}=-g_{i j} \partial_{r} \phi^{i} \partial_{r} \phi^{j} \\
-1+a^{2} b^{\prime 2}+\frac{a^{2^{\prime}} b^{2^{\prime}}}{2}=-\frac{1}{b^{2}} V_{\mathrm{eff}}\left(\phi_{i}\right)+a^{2} b^{2} g_{i j} \partial_{r} \phi^{i} \partial_{r} \phi^{j} \\
\partial_{r}\left(a^{2} b^{2} g_{i j} \partial_{r} \phi^{j}\right)=\frac{1}{2 b^{2}} \frac{\partial V_{\mathrm{eff}}}{\partial \phi^{i}}
\end{gathered}
$$

where' denotes derivatives with respect to $r$. The first three equations come from the Einstein equations and the last one is the equation of motion for the scalar. As discussed in Appendix A, $V_{\text {eff }}\left(\phi^{i}\right)$ is a function of scalars fields, $\phi^{i}$, and charges, $\left(Q_{A}, P^{A}\right)$ which is given by

$$
V_{\text {eff }}\left(\phi_{i}\right)=f^{A B}\left(Q_{A}-\tilde{f}_{A C} P^{C}\right)\left(Q_{B}-\tilde{f}_{B D} P^{D}\right)+f_{A B} P^{A} P^{B} .
$$

Modulo factors of $b^{2}$, one sees from (11) that $V_{\text {eff }}\left(\phi^{i}\right)$ is an "effective potential" for the scalar fields which is generated by non-trivial form fields. The effective potential, first discussed in [17], plays an important role in describing the attractor mechanism $[12,13,18,25,28,31,50]$.

\section{Non-extremal black holes}

In this section, we study spherically symmetric non-extremal black holes for a model with one scalar field non-minimally coupled to two gauge fields. ${ }^{7}$ In certain cases, with exponential couplings, the equations of motion can be solved exactly by rewriting them as Toda equations [23] (see Appendix B). Since we will consider exponential couplings, we need at least two terms in the effective potential it to have a minimum which we required for the attractor behaviour. Our solutions will be characterized by the mass, two gauge field charges, values of the moduli at infinity and the scalar charge. ${ }^{8}$

After discussing how to dress the charges and revisiting the equations of motion we will discuss some constraints on the charges which follow from evaluating the equations at the boundaries and then list some of the solutions. Finally, we discuss, in detail, the role of the scalar charge in the first law of thermodynamics for the simplest case from Sect. 3.4.

\footnotetext{
7 A subset of these models, with $\alpha_{1}=-\alpha_{2}$, are equivalent to a system with one gauge field with both electric magnetic charges turned on.

8 The scalar charge is not really an independent parameter-this is an example of secondary hair which is discussed further in Sect. 3.5 and 6.
} 
3.1 Scaling symmetry and dressing the charges

We consider two gauge fields with modulus dependent couplings of the form

$$
f_{A B}(\phi)=\delta_{A B} e^{\alpha_{B} \phi}
$$

for which exact solutions can be found. Given these couplings and taking the metric on the modulus to one, the matter Lagrangian is

$$
\mathcal{L}_{\text {matter }}=2(\partial \phi)^{2}+e^{\alpha_{1} \phi}\left(F_{1}\right)^{2}+e^{\alpha_{2} \phi}\left(F_{2}\right)^{2} .
$$

For electrically charged solutions, assuming the ansatz (7), the effective potential defined earlier becomes

$$
V_{\mathrm{eff}}=e^{-\alpha_{1} \phi}\left(Q_{1}\right)^{2}+e^{-\alpha_{2} \phi}\left(Q_{2}\right)^{2} .
$$

Alternatively for a dyonic black hole with a single gauge field we have

$$
V_{\mathrm{eff}}=e^{-\alpha_{1} \phi}\left(Q_{1}\right)^{2}+e^{\alpha_{1} \phi}\left(P_{1}\right)^{2} .
$$

The electric charges $Q_{A}$, can be written in terms of the following surface integral at spatial infinity

$$
Q_{A}=\oint_{S_{\infty}^{2}} * f_{A B} F^{B}
$$

From the equations of motion for the gauge fields, (5), one observes that this is the $U$ (1) charge one expects from Gauss' law modified in the presence of moduli. On the other hand the Lagrangian (14) is invariant under the global scaling symmetry

$$
\phi^{\prime}=\phi-\delta \phi, \quad F_{A}^{\prime}=e^{\alpha_{A} \delta \phi / 2} F_{A},
$$

but it is not hard to see that $Q_{A}$ is not invariant under this symmetry. However, one can define a dressed charge, $\bar{Q}_{A}$,

$$
\bar{Q}_{A}=e^{\frac{1}{2} \alpha_{A} \phi_{\infty}} \oint_{S_{\infty}^{2}} * f_{A B} F^{B}=Q_{A} e^{\frac{1}{2} \alpha_{A} \phi_{\infty}},
$$

which is invariant - the extra factor of $e^{\frac{1}{2} \alpha_{A} \phi_{\infty}}$ absorbs the change in $Q_{A}$. Similarly, for magnetic charges, we can define the dressed charge ${ }^{9}$

$$
\bar{P}_{A}=P_{A} e^{-\frac{1}{2} \alpha_{A} \phi_{\infty}}
$$

\footnotetext{
9 A simple mnemonic for remembering how the dressed charges are defined is to check what factors are required to keep the effective potential invariant under rescaling.
} 


\subsection{Equations of motion revisited}

Now, to recast the equations of motion as generalised Toda equations, and to facilitate some of the thermodynamic analysis, we define the following new variables

$$
u_{1}=\phi, \quad u_{2}=\log a, \quad z=\log a b, \quad “ . "=\partial_{\tau}=a^{2} b^{2} \partial_{r} .
$$

From the definition of $\tau$, we find

$$
r=\frac{r_{+} e^{-c \tau}-r_{-} e^{c \tau}}{e^{-c \tau}-e^{c \tau}}, \quad \text { where } c=\frac{1}{2}\left(r_{+}-r_{-}\right)
$$

and conversely

$$
\tau=\frac{1}{\left(r_{+}-r_{-}\right)} \log \left(\frac{r-r_{+}}{r-r_{-}}\right) .
$$

In terms of these new variables, the equations of motion become

$$
\begin{aligned}
\ddot{u}_{1} & =\frac{1}{2} \alpha_{1} e^{2 u_{2}+\alpha_{1} u_{1}} Q_{1}^{2}+\frac{1}{2} \alpha_{2} e^{2 u_{2}+\alpha_{2} u_{1}} Q_{2}^{2}, \\
\ddot{u}_{2} & =e^{2 u_{2}+\alpha_{1} u_{1}} Q_{1}^{2}+e^{2 u_{2}+\alpha_{2} u_{1}} Q_{2}^{2}, \\
\ddot{z} & =e^{2 z}, \\
\dot{z}^{2}-e^{2 z} & =\dot{u}_{1}^{2}+\dot{u}_{2}^{2}-e^{2 u_{2}+\alpha_{1} u_{1}} Q_{1}^{2}-e^{2 u_{2}+\alpha_{2} u_{1}} Q_{2}^{2} .
\end{aligned}
$$

The last equation is equivalent to the Hamiltonian constraint (10). The equation for $z$ (26), decouples from the other equations and is equivalent to (8). It can be used to show that the left hand side of the Hamiltonian constraint (27), is a constant, since

$$
\partial_{\tau}\left(\dot{z}^{2}-e^{2 z}\right)=2 \dot{z}\left(\ddot{z}-e^{2 z}\right) \stackrel{(26)}{=} 0 .
$$

So, letting $c^{2}=\dot{z}^{2}-e^{2 z}$, we can rewrite (27) as

$$
c^{2}={\dot{u_{1}}}^{2}+{\dot{u_{2}}}^{2}-e^{2 u_{2}+\alpha_{1} u_{1}} Q_{1}^{2}-e^{2 u_{2}+\alpha_{2} u_{1}} Q_{2}^{2} .
$$

The constant $c$ above, turns out to be the same as the one we defined in (22).

\subsection{Constraints on the charges}

Examining the equations of motion evaluated at the boundaries one finds two important properties of these black holes

$$
\begin{gathered}
M^{2}+g_{i j}\left(\phi_{\infty}\right) \Sigma^{i} \Sigma^{j}-V_{\mathrm{eff}}\left(\phi_{\infty}\right)=4 S^{2} T^{2}=c^{2} \\
2 g_{i j}\left(\phi_{\infty}\right) M \Sigma^{j}-2 g_{i j}\left(\phi_{\infty}\right) K^{j}-g_{i j, k}\left(\phi_{\infty}\right) \Sigma^{j} \Sigma^{k}=-\frac{1}{2} \frac{\partial V_{\mathrm{eff}}}{\partial \phi_{\infty}^{i}}
\end{gathered}
$$


where, $S$ and $T$ are the entropy and temperature of the black hole, and the scalar monopole and dipole charges, $\Sigma$ and $K$, are defined by the expansion of the moduli at infinity

$$
\phi^{j}=\phi_{\infty}^{j}+\frac{\Sigma^{j}}{r}+\frac{K^{j}}{r^{2}}+\cdots
$$

The first Eq. (30), is the Hamiltonian constraint (29) evaluated at the boundaries and it provides a constraint on charges. The second one (31), is an expression of the dependence of the scalar charges on the mass, the electric and magnetic charges and the values of the moduli at infinity. It follows from evaluating the scalar field equation at infinity.

For simplicity, we prove the relations (30) and (35), in the case of two charges and one scalar field, but the argument is easy to generalise. To prove (30), we evaluate the right hand side of (29) at spatial infinity and evaluate the constant $c^{2}$, at the horizon. We find that

$$
c^{2}=\dot{z}^{2}-e^{2 z}=\left[a b \partial_{r}(a b)\right]^{2}-e^{2 \ln (a b)}=\left(a a^{\prime} b^{2}\right)^{2}=4 S^{2} T^{2} .
$$

Here we used the fact that, for a non-extremal black hole with finite horizon area, $a(r)$ has a simple zero and $b$ is a constant at the horizon. In addition, we use the following expressions for the temperature and entropy

$$
T=\left.\frac{a a^{\prime}}{2 \pi}\right|_{r=r_{h}} \neq 0, \quad S=\pi b^{2}\left(r_{h}\right)
$$

Then, evaluating the right hand side of (29) at infinity gives the left hand side of (30). To prove (31), we consider the equation of motion for the scalar field (11), evaluated at infinity. Upon simplification, one finds, to first non-trivial order in $1 / r$

$$
\left[g_{i j}\left(1-\frac{2 M}{r}\right) r^{2}\left(\phi_{\infty}^{j}+\frac{\Sigma^{j}}{r}+\frac{K^{j}}{r^{2}}\right)^{\prime}\right]^{\prime}=\frac{1}{2 r^{2}} \frac{\partial V_{\mathrm{eff}}}{\partial \phi_{\infty}^{i}}
$$

which leads to (31).

\subsection{Exact solutions}

It is only for certain values of the parameters $\alpha_{A}$, that exact solutions are known. The parameter $\gamma$, given by

$$
\gamma=\frac{1}{2}\left(\sqrt{1-2 \alpha_{1} \alpha_{2}}-1\right)
$$

is useful for characterising the solutions: exact solutions are know for the cases $\gamma=$ $1,2,3[23,25]$. The details of constructing some exact non-extremal solutions are 
discussed in Appendix C. We merely list some of the solutions below for the cases $\gamma=1,2$.

\subsubsection{Case Ia: $\gamma=1$ and $\left|\alpha_{1,2}\right|=2$}

This solution has been extensively discussed in the literature. Since, in Sect. 3.5, we wish to discuss the role of both electric and magnetic charges in the first law, we present this solution as a dyonic black hole with a single gauge field so,

$$
V_{\mathrm{eff}}=e^{-2 \phi} Q^{2}+e^{2 \phi} P^{2} \text {. }
$$

The non-extremal solution is given by [27]:

$$
\begin{aligned}
\exp (2 \phi) & =e^{2 \phi_{\infty}} \frac{(r+\Sigma)}{(r-\Sigma)}, \\
a^{2} & =\frac{\left(r-r_{+}\right)\left(r-r_{-}\right)}{\left(r^{2}-\Sigma^{2}\right)}, \\
b^{2} & =\left(r^{2}-\Sigma^{2}\right),
\end{aligned}
$$

with

$$
r_{ \pm}=M \pm c, \quad c=\sqrt{M^{2}+\Sigma^{2}-\bar{Q}^{2}-\bar{P}^{2}}
$$

where we have defined the dressed charges $\bar{Q}=e^{\phi_{\infty}} Q$ and $\bar{P}=e^{-\phi_{\infty} P}$.

As already discussed, the scalar charge $\Sigma$, is not an independent parameter. It is given by

$$
\Sigma=\frac{\bar{P}^{2}-\bar{Q}^{2}}{2 M} .
$$

The extremal limit of the above solution corresponds to letting $c^{2} \rightarrow 0$ and the corresponding solution can be embedded in $\mathcal{N}=4$ supergravity.

\subsubsection{Case Ib: $\gamma=1, \sqrt{-\alpha_{1} \alpha_{2}}=2$ and $\alpha_{1}>\alpha_{2}$}

For this case,

$$
V_{\mathrm{eff}}=e^{-\alpha_{1} \phi} Q_{1}^{2}+e^{4 \phi / \alpha_{1}} Q_{2}^{2}
$$

The solution is

$$
\begin{aligned}
e^{\left(\alpha_{1}-\alpha_{2}\right) \phi} & =\left(\frac{1-\lambda}{\lambda}\right)\left(\frac{Q_{2} F_{2}}{Q_{1} F_{1}}\right)^{2} \\
a^{2} & =c^{2}\left(Q_{1} F_{1}\right)^{-2(1-\lambda)}\left(Q_{2} F_{2}\right)^{-2 \lambda} /\left\{\left(\frac{1-\lambda}{\lambda}\right)^{\lambda}+\left(\frac{1-\lambda}{\lambda}\right)^{1-\lambda}\right\} \\
b^{2} & =\left(r-r_{+}\right)\left(r-r_{-}\right) / a^{2}
\end{aligned}
$$


where

$$
F_{i}=\sinh \left(c\left(\tau-d_{i}\right)\right)
$$

and the ratio $\lambda$ is defined as

$$
\lambda=\frac{\alpha_{1}}{\alpha_{1}-\alpha_{2}} .
$$

Notice that $\lambda$ lies between 0 and 1 . The integration constants $c, r_{ \pm}$, and $d_{i}$ are given in terms of $M, \Sigma$, and the "dressed" charges

$$
\bar{Q}_{i}^{2}=e^{\alpha_{i} \phi_{\infty}} Q_{i}^{2}
$$

by

$$
\begin{aligned}
& c^{2}=M^{2}+\Sigma^{2}-\bar{Q}_{1}^{2}-\bar{Q}_{2}^{2} \\
& r_{ \pm}=M \pm c \\
& \sinh ^{2}\left(c d_{i}\right)=\left[\frac{4 c^{2}}{\alpha_{i}^{2}+4}\right] \bar{Q}_{i}^{-2} .
\end{aligned}
$$

Due to the fact that the parameters $\alpha_{1}$ and $\alpha_{2}$ are very weakly constrained, we find it unlikely that all solutions in this class could be embedded in supergravity theory.

3.4.3 Case II: $\gamma=2$ and $\left|\alpha_{1,2}\right|=2 \sqrt{3}$

This case arises from the Kaluza-Klein reduction of the $5 d$ Schwarzschild black hole so it is natural to write it as a dyonic solution with

$$
V_{\mathrm{eff}}=e^{-2 \phi / \sqrt{3}} Q^{2}+e^{2 \phi / \sqrt{3}} P^{2} \text {. }
$$

The solution can be written as [14,24]

$$
\begin{aligned}
\exp (4 \phi / \sqrt{3}) & =e^{4 \phi_{\infty} / \sqrt{3}} \frac{A}{B} \\
a^{2} & =\frac{\left(r-r_{+}\right)\left(r-r_{-}\right)}{\sqrt{A B}} \\
b^{2} & =\sqrt{A B} \\
A & =\left(r-r_{A_{+}}\right)\left(r-r_{A_{-}}\right) \\
B & =\left(r-r_{B_{+}}\right)\left(r-r_{B_{-}}\right) \\
r_{ \pm} & =M \pm \sqrt{M^{2}+\Sigma^{2}-\bar{P}^{2}-\bar{Q}^{2}}=M \pm c \\
r_{A_{ \pm}} & =\frac{1}{\sqrt{3}} \Sigma \pm \bar{P} \sqrt{\frac{2 \Sigma}{\Sigma-\sqrt{3} M}}
\end{aligned}
$$




$$
\begin{aligned}
r_{B_{ \pm}} & =-\frac{1}{\sqrt{3}} \Sigma \pm \bar{Q} \sqrt{\frac{2 \Sigma}{\Sigma+\sqrt{3} M}} \\
\text { Area } & =4 \pi \sqrt{\left(r_{+}-r_{A_{+}}\right)\left(r_{+}-r_{A_{-}}\right)\left(r_{+}-r_{B_{+}}\right)\left(r_{+}-r_{B_{-}}\right)}
\end{aligned}
$$

where we have defined the dressed charges $\bar{Q}=e^{\frac{1}{\sqrt{3}} \phi_{\infty}} Q$ and $\bar{P}=e^{-\frac{1}{\sqrt{3}} \phi_{\infty}} P$.

Once again $\Sigma$ is not an independent parameter and is given by,

$$
\frac{2}{\sqrt{3}} \Sigma=\frac{\bar{Q}^{2}}{\sqrt{3} M+\Sigma}-\frac{\bar{P}^{2}}{\sqrt{3} M-\Sigma} .
$$

In the extremal limit ( $c=0$ ) we obtain a non-BPS black hole that can be embedded in $\mathcal{N}=2$ supergravity.

\subsection{Scalar charge and the first law of thermodynamics}

We have seen previously that, unlike in the case of minimally-coupled scalars, ${ }^{10}$ the black hole solutions we consider carry scalar charge. It is important to mention that the scalar charge is not protected by a gauge symmetry, and hence is not a conserved charge.

In the cases we studied, the scalar charge is not an independent parameter. It depends on the other asymptotic charges, namely the ADM mass and the dressed gauge field charges. This implies that just one of the parameters $\phi_{\infty}$ and $\Sigma$ is independent. This kind of scalar charge, which depends on other asymptotic data, is called secondary hair. As it depends on the gauge field charges, it does not represent a new quantum number associated with the black hole.

Due to the non-minimal coupling of the scalar fields, the first law gets modified. It should be supplemented by a new term containing the variation of the moduli [22]

$$
d M=T d S+\psi^{A} d Q_{A}+\psi_{A} d P^{A}-\Sigma_{i} d \phi_{\infty}^{i}
$$

where $\left(\psi^{A}, \psi_{A}\right)$ are the potentials conjugate to the charges $\left(Q_{A}, P^{A}\right)$.

Indeed, we will show explicitly that this is the case for some of the black hole solutions considered here. We need to check that $M\left(S, Q_{A}, P^{A}, \phi_{\infty}\right)$ is an exact differential. Since it is particular to this class of black holes, we are mainly interested in the non-trivial term $\Sigma_{i}=-\left.\left(\partial M / \partial \phi_{\infty}^{i}\right)\right|_{\left(S, Q_{A}, P^{A}\right)}$, but similar computations can be done for the other terms. ${ }^{11}$

While we have verified the first law for all the solutions in the previous section, we present, in detail, the analysis of the solution (Ia), which has $\gamma=1$ and $\alpha_{1}=-\alpha_{2}=2$, from Sect. 3.4.1. In that case, there are two conserved gauge charges $(P, Q)$ and a

\footnotetext{
10 For minimally-coupled scalars the standard no-hair theorems apply and do not allow for a nice solution with non-zero scalar charge.

11 Note that, requiring cosmic censorship imposes the condition $M \geq \Sigma$ since, there is a curvature singularity at $r=\Sigma$ while the outer horizon is at $r_{+}=M+c$ (which approaches $M$ as $c \rightarrow 0$ ).
} 
scalar charge. We rewrite some of the equations from Sect. 3.4.1, as well as the entropy, in the following useful way:

$$
\begin{aligned}
2 M \Sigma & =\bar{P}^{2}-\bar{Q}^{2}, \\
c & =\sqrt{\left(M+\frac{\bar{P}^{2}-\bar{Q}^{2}}{2 M}\right)^{2}-2 \bar{P}^{2}}, \\
S & =\pi b^{2}\left(r_{+}\right)=\pi\left[(c+M)^{2}-\Sigma^{2}\right]=\pi\left(2 M^{2}-\bar{Q}^{2}-\bar{P}^{2}+2 c M\right) .
\end{aligned}
$$

Now, by differentiating (61) and (62) at fixed entropy and charge parameters $Q, P$, we obtain:

$$
\begin{aligned}
d c & =\frac{1}{c}(M+\Sigma)\left(1-\frac{\Sigma}{M}\right) d M+\frac{1}{c}\left[2 \bar{P}^{2}-\frac{M+\Sigma}{M}\left(\bar{P}^{2}+\bar{Q}^{2}\right)\right] d \phi_{\infty}, \\
0 & =4 M d M-2 \bar{Q}^{2} d \phi_{\infty}+2 \bar{P}^{2} d \phi_{\infty}+2 c d M+2 M d c .
\end{aligned}
$$

The next step is to use (63) in (64) and to check that, indeed, $\Sigma=-\left.\left(\partial M / \partial \phi_{\infty}\right)\right|_{S, Q, P}$. We can calculate the other intensive parameters in the same way and we obtain the following expressions:

$$
\psi^{Q}=\frac{\bar{Q}^{2}}{Q} \frac{M+\Sigma+c}{(M+c)^{2}-\Sigma^{2}}, \quad \psi^{P}=\frac{\bar{P}^{2}}{P} \frac{M+c-\Sigma}{(M+c)^{2}-\Sigma^{2}} .
$$

With all these expressions one can easily check that the first law is satisfied

$$
d M=T d S+\psi^{Q} d Q+\psi^{P} d P-\Sigma_{i} d \phi_{\infty}^{i}
$$

The fact that the Lagrangian has a global scaling symmetry (18), suggests writing the first law in terms of the "dressed" charges:

$$
d M=T d S+\bar{\psi}^{Q} d \bar{Q}+\bar{\psi}^{P} d \bar{P}
$$

where $\bar{\psi}^{Q}$ and $\bar{\psi}^{P}$ are the conjugate potentials of the dressed charges $\bar{Q}$ and $\bar{P}$, respectively. One can again compute the values of the intensive parameters as above or one can rewrite (67) as

$$
d M=T d S+\bar{\psi} Q \frac{\bar{Q}}{Q} d Q+\bar{\psi}^{P} \frac{\bar{P}}{P} d P+\left(\bar{\psi}^{Q} \bar{Q}-\bar{\psi}^{P} \bar{P}\right) d \phi_{\infty}
$$

and then compare with (66). One obtains the following expressions for the intensive parameters

$$
\bar{\psi}^{Q}=\frac{\bar{Q}}{M+c-\Sigma}, \quad \bar{\psi}^{P}=\frac{\bar{P}}{M+c+\Sigma} .
$$


As an application of our formulae consider what happens if we add some scalar particles to the black hole. We keep $\bar{Q}$ and $\bar{P}$ (or equivalently $Q, P$ and $\phi_{\infty}$ ) fixed. Taking a differential of (60) and using the first law with $\bar{Q}$ and $\bar{P}$ constant gives

$$
\delta S=-\frac{M}{T} \frac{\delta \Sigma}{\Sigma}
$$

From (70) we see that increasing $|\Sigma|$ (i.e. when $d \Sigma / \Sigma>0$ ) causes the entropy to decrease. This implies that adding scalar charge induces Hawking radiation. Conversely, reducing $|\Sigma|$ simply causes the black hole to puff up.

Note that (67) does not involve a variation with respect to the asymptotic value of the scalars. However, let us recall that the physical conserved charges (due to the equations of motion) are $Q_{A}$ given in (17) and so the scaling symmetry does not preserve the conserved charges. By making a scaling one can generate new solutions. However, the new solution can not be reached dynamically starting from the old one because this will also force a violation of charge conservation. To obtain the first law in this form, one should supplement the quasilocal formalism by a boundary counterterm that depends of moduli- this term is taking care of the non-conserved charge in the first law.

\section{Attractor mechanism}

In this section, we discuss the attractor mechanism using both, the effective potential (12) method [25] and the entropy function [44] framework. The first method is based on investigating the equations of motion of the moduli and finding the conditions satisfied by the effective potential such that the attractor phenomenon occurs. We will extend the calculations of [25] for non-extremal black holes. The entropy function approach focuses on the near-horizon geometry and its enhanced symmetries. The equivalence of the effective potential approach and entropy function formalism in the context of four-dimensional extremal non-BPS black hole solutions in $\mathcal{N}=2$ supergravity has recently been discussed in [6]. In the last section, we shall briefly mention the relevant aspects of the attractor phenomenon in special geometry [17,29].

\subsection{Effective potential and non-supersymmetric attractors}

We consider again the solution (Ia) and we investigate its extremal limit, $c \rightarrow 0$. We use (60)-(62) and in the extremal limit we obtain:

$$
\begin{aligned}
2 M \Sigma & =\bar{P}^{2}-\bar{Q}^{2}, \\
0 & =\sqrt{\left(M+\frac{\bar{P}^{2}-\bar{Q}^{2}}{2 M}\right)^{2}-2 \bar{P}^{2}}=\sqrt{(M+\Sigma)^{2}-2 \bar{P}^{2}}, \\
S & =\pi b^{2}\left(r_{+}\right)=\pi\left(M^{2}-\Sigma^{2}\right) .
\end{aligned}
$$


One can easily solve the system of the first two equations to obtain $M$ and $\Sigma$ as functions of the dressed charges $\bar{Q}=e^{\phi_{\infty}} Q$ and $\bar{P}=e^{-\phi_{\infty}} P$. Then, the mass and the scalar charge depend on the asymptotic values of the moduli $\phi_{\infty}$ as follows,

$$
\begin{aligned}
& M=\frac{1}{\sqrt{2}}(\bar{P}+\bar{Q}), \\
& \Sigma=\frac{1}{\sqrt{2}}(\bar{P}-\bar{Q}) .
\end{aligned}
$$

However, the entropy becomes independent of $\phi_{\infty}$, i.e.

$$
S=2 \pi P Q
$$

The entropy is also independent of moduli for the other solutions. In what follows, we briefly review the effective potential method of [25] to clarify these interesting results.

For the attractor phenomenon to occur, it is sufficient if the following two conditions are satisfied [25]. First, for fixed charges, as a function of the moduli, $V_{\text {eff }}$ must have a critical point. Denoting the critical values for the scalars as $\phi^{i}=\phi_{0}^{i}$ we have,

$$
\partial_{i} V_{\text {eff }}\left(\phi_{0}^{i}\right)=0
$$

Second, there should be no unstable directions about this minimum, so the matrix of second derivatives of the potential at the critical point,

$$
M_{i j}=\frac{1}{2} \partial_{i} \partial_{j} V_{\mathrm{eff}}\left(\phi_{0}^{k}\right)
$$

should have no negative eigenvalues. Schematically we can write,

$$
M_{i j}>0
$$

The eigenvalues of $M_{i j}$ are proportional to the effective mass squared for the fields, $\phi^{i}$, near the attractor point.

We can consistently set the moduli to constants if we fix them at their critical values. The theory effectively reduces to Einstein-Maxwell gravity which has the extremal Reissner-Nordstrom black hole as a solution. If we then examine what happens if the asymptotic value of the asymptotic moduli deviate slightly from the attractor value, simultaneously demanding that the black hole remains extremal (that is the horizon still has a double zero), one finds attractor behaviour: the moduli attain their critical values at the horizon and entropy remains independent of the value of the moduli at infinity [25]. The horizon radius is given by

$$
b_{H}^{2}=V_{\mathrm{eff}}\left(\phi_{0}^{i}\right),
$$


and the entropy is

$$
S_{B H}=\frac{1}{4} A=\pi b_{H}^{2}=\pi V_{\mathrm{eff}}\left(\phi_{0}^{i}\right)
$$

Now, one can verify the attractor behaviour for our effective potential given in (15). The condition for the existence of an extremum for the effective potential will give us the value of the moduli at the horizon

$$
0=\frac{\partial V_{\mathrm{eff}}}{\partial \phi}=\alpha_{1} e^{\alpha_{1} \phi}\left(Q_{1}\right)^{2}+\alpha_{2} e^{\alpha_{2} \phi}\left(Q_{2}\right)^{2} \Longrightarrow e^{\left(\alpha_{1}-\alpha_{2}\right) \phi_{0}}=-\frac{\alpha_{2}}{\alpha_{1}} \frac{Q_{2}^{2}}{Q_{1}^{2}} .
$$

Then, the value of the moduli at the horizon depends just on the charge parameters and not on the boundary conditions. It is a simple exercise to check that the second derivative of the potential is positive and hence the extremum is a minimum. For $\alpha_{1} \neq-\alpha_{2}$ with $\alpha_{1} \alpha_{2}=-4$ the entropy is

$$
S=\pi V_{\mathrm{eff}}\left(\phi_{0}\right)=\pi Q_{1}^{\frac{8}{\alpha_{1}^{2}+4}} Q_{2}^{\frac{2 \alpha_{1}^{2}}{\alpha_{1}^{2}+4}}\left[\left(\frac{4}{\alpha_{1}^{2}}\right)^{\frac{\alpha_{1}^{2}}{\alpha_{1}^{2}+4}}+\left(\frac{4}{\alpha_{1}^{2}}\right)^{-\frac{4}{\alpha_{1}^{2}+4}}\right]
$$

The same result is obtained by taking the extremal limit $c=0$ in the non-extremal entropy (167). For $\alpha_{1}=-\alpha_{2}=2$, the entropy is given by

$$
S=\pi V_{\mathrm{eff}}\left(\phi_{0}\right)=2 \pi Q_{1} Q_{2},
$$

or for a dyonic black hole

$$
S=2 \pi P Q
$$

It is important to note that in deriving the conditions for the attractor phenomenon, one does not have to use supersymmetry at all. We will obtain the same result in the next section by using the entropy function.

\subsection{Entropy function}

The near-horizon geometry of the extremal charged black holes has been shown to have a geometry of $A d S_{2} \times S^{2}$ and, when embedded in certain supergravities, has an enhanced supersymmetry.

As has been discussed in [16,29], the moduli do not preserve any memory of the initial conditions at infinity due to the presence of the infinite throat of $A d S_{2}$. This is in analogy with the properties of the behavior of dynamical flows in dissipative systems, where, on approaching the attractors, the orbits practically lose all the memory of their initial conditions. 
Let us investigate the near-horizon geometry of non-extremal spherically symmetric black holes, where the line element is given by,

$$
d s^{2}=-a(r)^{2} d t^{2}+a(r)^{-2} d r^{2}+b(r)^{2} d \Omega^{2}
$$

The Einstein equation (8), $\left(a^{2} b^{2}\right)^{\prime \prime}=2$, can be integrated out and one gets $a^{2} b^{2}=$ $\left(r-r_{+}\right)\left(r-r_{-}\right)$. The interpretation of the parameters $r_{+}$and $r_{-}$is that they are related to the outer and the inner horizon, respectively. Next, we introduce the non-extremality parameter $\epsilon$ and also make a change of coordinates such that the horizon is at $\rho=0$, i.e.

$$
\rho=r-r_{+}, \quad \epsilon=r_{+}-r_{-} .
$$

The extremal black hole is obtained when the inner and the outer horizons coincide. For the non-extremal solution $\left(r_{+} \neq r_{-}\right)$, we have,

$$
a^{2} b^{2}=\rho(\rho+\epsilon)
$$

Let us take,

$$
\begin{aligned}
& a^{2}=\rho f(r)=\rho\left(f_{0}+f_{1} \rho+f_{2} \rho^{2}+\cdots\right), \\
& b^{2}=\frac{\rho(\rho+\epsilon)}{a^{2}}=\frac{\rho+\epsilon}{f_{0}+f_{1} \rho+f_{2} \rho^{2}+\cdots},
\end{aligned}
$$

where, $f(r)$ has been expanded as a power series in $\rho$. The near-horizon geometry is obtained by taking the limit $\rho \rightarrow 0$ and is given by

$$
d s^{2}=-\left(\rho f_{0}\right) d t^{2}+\frac{1}{\rho f_{0}} d \rho^{2}+\frac{\epsilon}{f_{0}} d \Omega^{2} .
$$

The temperature and the entropy of the non-extremal black hole are given by

$$
T=\left.\frac{\left(a^{2}\right)^{\prime}}{4 \pi}\right|_{\rho=0}, \quad S=\left.\frac{4 \pi b^{2}}{4}\right|_{\rho=0} .
$$

By comparing these expressions with the expressions obtained from the near-horizon geometry (91), one can read off the following expressions for the parameters appearing in (91):

$$
f_{0}=4 \pi T, \quad \epsilon=\frac{f_{0} S}{\pi}=4 T S=2 c .
$$

To see that the near-horizon geometry of the extremal solution is $A d S_{2} \times S^{2}$, we take the extremal limit $T \sim f_{0} \rightarrow 0$ and expand the metric to first non-trivial order about 
$\rho=0$. It is not hard to see that this procedure gives:

$$
d s^{2}=\frac{1}{f_{1}}\left(-\rho^{2} d t^{2}+\frac{1}{\rho^{2}} d \rho^{2}\right)+\frac{1}{f_{1}} d \Omega^{2},
$$

where we have rescaled the time variable: $t \rightarrow t / f_{1}$.

It is crucial that the near horizon geometry is $A d S_{2} \times S^{2}$ : Sen's entropy function formalism, [44], assumes from the beginning that the metric and all other fields respect the $S O(2,1) \times S O(3)$ symmetry of $A d S_{2} \times S^{2} .^{12}$

In [44], it was observed that the entropy of a spherically symmetric extremal black hole is given by the extremum of the Legendre transform (with respect to the electric field) of the Lagrangian density evaluated at the horizon. The derivation of this result does not require that the theory or the solution are supersymmetric. The only requirements are gauge and general coordinate invariance of the action and the assumption that the near horizon geometry is $A d S_{2} \times S^{2}$.

The entropy function is defined as

$$
\begin{aligned}
\mathcal{E}(\vec{u}, \vec{v}, \vec{e}, \vec{p}) & =2 \pi\left(e_{i} q_{i}-f(\vec{u}, \vec{v}, \vec{e}, \vec{p})\right) \\
& =2 \pi\left(e_{i} q_{i}-\int_{H} d \theta d \phi \sqrt{-G} \mathcal{L}\right),
\end{aligned}
$$

where $q_{i}=\partial f / \partial e_{i}$ are the electric charges, $u_{s}$ are the values of the moduli at the horizon, $p_{i}$ and $e_{i}$ are the near horizon radial magnetic and electric fields and $v_{1}, v_{2}$ are the sizes of $A d S_{2}$ and $S^{2}$, respectively. Thus, $\mathcal{E} / 2 \pi$ is the Legendre transform of the reduced Lagrangian $f$, with respect to the variables $e_{i}$. For an extremal black hole of electric charge $\vec{Q}$ and magnetic charge $\vec{P}$, Sen has shown that the equations determining $\vec{u}, \vec{v}$, and $\vec{e}$ are given by

$$
\frac{\partial \mathcal{E}}{\partial u_{s}}=0, \quad \frac{\partial \mathcal{E}}{\partial v_{i}}=0, \quad \frac{\partial \mathcal{E}}{\partial e_{i}}=0 .
$$

Then, the black hole entropy is given by $S=\mathcal{E}(\vec{u}, \vec{v}, \vec{e}, \vec{p})$ at the extremum (96). The entropy function, $\mathcal{E}(\vec{u}, \vec{v}, \vec{e}, \vec{p})$, determines the sizes $v_{1}, v_{2}$ of $A d S_{2}$ and $S_{2}$ and also the near horizon values of moduli $u_{s}$ and gauge field strengths $e_{i}$. If $\mathcal{E}$ has no flat directions, then the extremization of $\mathcal{E}$ determines $\vec{u}, \vec{v}, \vec{e}$ in terms of $\vec{Q}$ and $\vec{P}$. Therefore, $S=\mathcal{E}$ is independent of the asymptotic values of the scalar fields. These results lead to a generalised attractor phenomenon for both supersymmetric and non-supersymmetic extremal black hole solutions.

Now we can apply this method to our action (2) with a zero axionic coupling. We are interested in a theory with one scalar field and one electromagnetic field with both

\footnotetext{
12 In the rotating case, due to the axial symmetry, the $S O(3)$ symmetry is broken to a $U(1)$ symmetry. However, the long throat of $A d S_{2}$ is still present and the entropy function formalism, slightly modified, can still be applied [2].
} 
electric and magnetic charges turned on

$$
S=\frac{1}{\kappa^{2}} \int d^{4} x \sqrt{-G}\left(R-2(\partial \phi)^{2}-e^{2 \phi} F^{2}\right) .
$$

The general metric of $A d S_{2} \times S^{2}$ can be written as

$$
d s^{2}=v_{1}\left(-\rho^{2} d t^{2}+\frac{1}{\rho^{2}} d \rho^{2}\right)+v_{2}\left(d \theta^{2}+\sin ^{2} \theta d \phi^{2}\right) .
$$

The field strength ansatz (7) in our case is given by

$$
F=e d t \wedge d r+P \sin \theta d \theta \wedge d \phi=e^{-2 \phi} Q d t \wedge d r+P \sin \theta d \theta \wedge d \phi
$$

The entropy function, $\mathcal{E}\left(v_{1}, v_{2}, e, q, p\right)$, and reduced Lagrangian, $f\left(v_{1}, v_{2}, e, p\right)$, are given by

$$
\begin{aligned}
\mathcal{E}\left(v_{1}, v_{2}, e, q, p\right) & =2 \pi\left[q e-f\left(v_{1}, v_{2}, e, p\right)\right], \\
f\left(v_{1}, v_{2}, e, p\right) & =\frac{8 \pi}{k^{2}}\left[-v_{2}+v_{1}-e^{2 \phi}\left(\frac{-v_{2}}{v_{1}} e^{2}+\frac{v_{1}}{v_{2}} P^{2}\right)\right] .
\end{aligned}
$$

Then the attractor equations are obtained as

$$
\begin{aligned}
& \frac{\partial \mathcal{E}}{\partial v_{1}}=0 \Rightarrow 1-\frac{v_{2}}{v_{1}^{2}} e^{2 \phi} e^{2}-\frac{1}{v_{2}} e^{2 \phi} P^{2}=0 \\
& \frac{\partial \mathcal{E}}{\partial v_{2}}=0 \Rightarrow-1+\frac{1}{v_{1}} e^{2 \phi} e^{2}-\frac{v_{1}}{v_{2}^{2}} e^{2 \phi} P^{2}=0 \\
& \frac{\partial \mathcal{E}}{\partial \phi}=0 \Rightarrow\left(P^{2}-e^{2}\right)=0 \\
& \frac{\partial \mathcal{E}}{\partial e}=0 \Rightarrow q=\frac{16 \pi}{k^{2}}\left(\frac{v_{2}}{v_{1}} e^{2 \phi} e\right)
\end{aligned}
$$

By combining the first two equations we obtain, $v=v_{1}=v_{2}=e^{2 \phi}\left(e^{2}+P^{2}\right)$, which is also expected from our near horizon geometry analysis as discussed before. The third equation gives the value of the moduli at the horizon $e^{-4 \phi}=P^{2} / Q^{2}$ and therefore, $v=2 P Q$. Now one can check that the entropy is given by the value of the entropy function $\mathcal{E}$ evaluated at the attractor point

$$
S=\mathcal{E}=2 \pi P Q=\pi v
$$

Using the electromagnetic field ansatz, one can show that $S=\pi V_{\text {eff }}$ and $q=-Q$ (negative sign appears because of our convention for $F_{t r}$ ). 
Before going on to discuss how the attractor behaviour breaks down for non-extremal black holes, we consider the analogy between dissipative dynamical systems, their attractor behaviour and black hole attractors.

Dissipative dynamical systems are characterized by the presence of some sort of internal "friction" that tends to contract the phase-space volume elements. Attractors are states towards which a system starting from certain initial conditions may evolve after a long enough time. Attractors can be unique states, called fixed point attractors. ${ }^{13}$

Now, the extremal black hole attractors sit at the bottom of a infinitely deep $A d S_{2}$ throat. The authors of [29], draw an analogy between the radial evolution of the scalar field down the throat and an under-damped oscillator, which, given sufficient time will settle to its equilibrium position independent of the initial conditions. Dissipation erases the "memory" of the initial conditions.

Like any analogy, this one has its uses and short comings. If one examines the differential equations which describe the radial evolution of the scalars and the metric, one finds they have to be fine tuned to get the extremal solution. The equations can be mapped to a mechanical model of a ball rolling up a hill. The radial parameter maps to time and the initial position and velocity of the ball can be mapped to the asymptotic values of the scalar and scalar charge. For the attractor black hole solution, the ball comes to rest precisely at the top. Any trajectory which does not come to rest at the top corresponds to the scalars blowing up at the horizon which is unphysical. Clearly one has to chose the initial velocity of the ball rather judiciously to obtain the solution-from the perspective of the mechanical model, this is the complete opposite of attractor behaviour. However, the point is that this mechanical model does not capture all the physics of the situation.

First the fine-tuning of the ball's initial velocity, physically corresponds to the fact that, as we have previously seem, the scalar charge is not an independent parameter. We should not find this too disturbing since the scalar charge is not a conserved quantity.

Secondly, once we consider how our black hole might form we see that it does indeed display conventional attractor behaviour-that is the final state does not depend on initial conditions. Consider an arbitrary distribution of matter which collapses to form a blackhole with certain gauge charges. Generically, such a collapse would produce a non-extremal black hole. This blackhole would then cool via Hawking radiation and approach extremality. Since there is only one extremal solution-the attractor solution-it much approach this solution as the black hole cools. As it cools, the throat becomes deeper and deeper and the black hole becomes more and more sequestered from its environment eventually completely forgetting about its initial conditions. This is directly analogous to an under-damped oscillator. Once we invoke semi-classical effects, we see that the attractor black hole is attractive in the conventional sense-

\footnotetext{
13 For linear dissipative dynamical systems, fixed point attractors are the only possible type of attractor. Nonlinear systems, on the other hand, harbor a much richer spectrum of attractor types. For example, in addition to fixed-points, there may exist periodic attractors such as limit cycles. There is also an intriguing class of chaotic attractors called strange attractors that have a complicated geometric structure.
} 
given generic initial conditions (that is some arbitrary matter distribution), we expect to end up with the attractor solution.

In using this analogy, one should be careful about how one relates radial evolution to time. We can associate the radial direction with time evolution, since, as the black hole cools, the throat becomes deeper. This should not be confused with the map between the radial parameter and the time in an artificial mechanical model.

It is amusing to contrast the fine tuning of the mechanical model with the genericity of the attractor solution we argue for above. Perhaps this fine tuning corresponds to the fine tuning that would be required to form an extremal black hole without invoking semi-classical effects.

Finally, we note that there may be flat directions in the entropy function. This can lead to generalised attractor behaviour in which the entropy is independent of the moduli but the near horizon geometry is not [2]. Extending our analogy, one might suppose that flat directions correspond to modes that do not couple to the dissipation and can persist even in the extremal limit. The black hole forgets enough to ensure the entropy does not depend on the moduli. ${ }^{14}$

\subsection{Non-extremal solutions and unattractor equation}

We would like to understand the relation between the entropy and the value of effective potential at the horizon for the non-extremal black holes. The first observation is that the near-horizon geometry of a non-extremal black hole (91) does not contain an $A d S_{2}$ part. Since, as we have seen, the $A d S_{2}$ symmetries implied the attractor behaviour it is plausible to suppose that the converse applies-in the absence of these symmetries there is no attractor behaviour. Then, the black hole horizon is not an attractor for the moduli. The effective potential evaluated at the horizon and the entropy will receive corrections away from the attractor value which depend on the asymptotic values of the moduli.

We investigate (10) and (11) at the horizon. Using some results from Sect. 4.2, namely,

$$
f_{0}=4 \pi T, \quad \epsilon=\frac{f_{0} S}{\pi}=4 T S=2 c .
$$

we try to write things in terms of the temperature and entropy as much as possible. Equation (10) gives us a relation between the entropy and the value of the effective potential at the horizon

$$
V_{\mathrm{eff}}=\frac{\epsilon}{2 f_{0}}\left(1+\frac{\epsilon f_{1}}{f_{0}}\right)=\frac{S}{2 \pi}\left(1+\frac{S f_{1}}{\pi}\right) .
$$

\footnotetext{
14 Notwithstanding information leaking through the deep throat, on might say that the generalised black hole attractor behaves like an ideal "tricky" politician, choosing to forget (or erase) only compromising details.
} 
The other Eq. (11), evaluated at the horizon gives us

$$
\frac{\partial V_{\mathrm{eff}}}{\partial \phi}=\frac{S^{2}}{\pi^{2}} \sqrt{2 f_{0} f_{2}+\frac{1}{2}\left(\frac{\pi}{S}-f_{1}\right)\left(\frac{\pi}{S}+3 f_{1}\right)} .
$$

There is a class of near-extremal black holes which break supersymmetry, but whose entropy can still be accounted for by microscopic counting [5,26]. These are fivedimensional black hole solutions and in the extremal limit, the near-horizon geometry contains an $A d S_{3}$ factor, rather than an $A d S_{2}$. In this case one can use the $A d S_{3} / C F T_{2}$ correspondence and the Cardy formula to compute the entropy. Unfortunately, there is no entropy function formalism for this kind of black holes. However, it was pointed out in [42] that there is a nice relation between $A d S_{3}$ and $A d S_{2}$ in the context of attractor mechanism. Also, Maldacena [35] observed that the supersymmetry of the theory describing the excitations of the D-branes is similar to $\mathcal{N}=2$ in four dimensions, the supersymmetry we are interested in. That is an $(1+1)$-dimensional field theory with $(4,4)$ susy - this is the susy left unbroken by the extremal D-branes. There are vector multiplets and hypermultiplets and the distinction between them is that they have different transformation properties under $R$ symmetries.

Let us comment on the role of the effective potential in the case of near-extremal black holes. We saw that, in the extremal case, the fixed $t$-surface takes the geometry of an infinite cylinder (the "infinite throat"). It seems that the horizon has been pushed away to infinity, though one can still fall into the black hole in finite proper time since the horizon is still a finite distance away in time-like or null directions. The near horizon geometry of the non-extremal black hole is rather similar to Rindler space as opposed to $A d S_{2} \times S^{2}$. In this case, the effective potential is not generically extremised and the attractor behaviour is absent. However, there is a special case when the attractor is still useful. Let us consider black holes with large charges $(Q \gg 1)$. Now, let us repeat the arguments of [35] to explain the microscopic/macroscopic agreement for the near-extremal black holes. In our discussion, we can keep $g_{s}$ small (closed string effects are small) and we obtain a strong coupling regime because of the large number $Q$ of branes: the fundamental strings couple weakly to each other but interact strongly with the collection of D-branes. The effective open string coupling is $g_{s} Q$. For $g_{s} Q \ll 1$ we obtain the domain of validity of the D-brane perturbation theory and for $g_{s} Q \gg 1$ we obtain the semi-classical black hole domain.

Using our Eq. (107), one finds that, in the near-extremal limit and for large charges, the entropy is still given by the value of the effective potential at the horizon. In the near-extremal limit, the effective potential depends on the values of moduli at infinity. However, the string coupling is small and so the corrections received by the effective potential are small in comparison with its value in the extremal limit. The near-horizon geometry is approximately $A d S_{2} \times S^{2}$ and the attractor mechanism still works in this case.

\subsection{First order perturbation analysis of non-extremal black holes}

To put our discussion of non-extremal unattractive black holes on a more quantitative footing, we study the effect of perturbing a non-extremal black hole away from 
the attractor point. We will start with a non-extremal Reissner-Nordstrom black hole where the scalar field is fixed at the attractor value everywhere. We ask how the solution changes if we shift the asymptotic value of the scalar slightly away from the attractor point. In particular we are interested in how the value of the scalar field changes at the horizon. This extends the perturbation analysis of [25] which mainly considered extremal black holes. ${ }^{15}$

For concreteness we consider an effective potential of the form

$$
V_{\mathrm{eff}}=e^{-\alpha \phi} Q^{2}+e^{\alpha \phi} P^{2}
$$

We can consistently set the scalar, $\phi$, to a constant if it is at the attractor point, $\partial_{\phi} V_{\text {eff }}=0$. Extremising the effective potential one finds

$$
e^{2 \alpha \phi_{0}}=Q^{2} / P^{2}
$$

where $\phi_{0}$ is the attractor value. Now, with the scalar constant everywhere, we effectively have Einstein-Maxwell gravity which in particular has the non-extremal Reissner-Nordstrom black hole as a solution.

We now perturb the non-extremal Reissner-Nordstrom black hole by assuming that the scalar varies slightly from the attractor value

$$
\phi(r)=\phi_{0}+\varepsilon \phi_{1}(r)
$$

Here $\varepsilon$ is a small parameter we use to organise the perturbation theory. As discussed in [25], the scalar perturbation (111), will source second order perturbations to the metric. This back-reaction will in turn source second order perturbations to the scalar field etc.

Let us write

$$
\begin{aligned}
a^{2} & =a_{0}^{2}+\varepsilon^{2} a_{2} \\
b & =b_{0}+\varepsilon^{2} b_{2}
\end{aligned}
$$

where $b_{0}$ and $a_{0}$ are the unperturbed non-extremal Reissner-Nordstrom solution. They are given by,

$$
\begin{aligned}
& a_{0}^{2}=\frac{1}{r^{2}}\left(r-r_{+}\right)\left(r-r_{-}\right) \\
& b_{0}=r
\end{aligned}
$$

with $r_{ \pm}=M \pm \sqrt{M^{2}-\left(\bar{Q}^{2}+\bar{P}^{2}\right)}=M \pm \sqrt{M^{2}-2|Q P|}$.

\footnotetext{
15 While the non-extremal case was discussed in [25], the first order analysis was only done approximately. Our results confirm the qualitative picture discussed there.
} 
Now, expanding the equation for the scalar field, (4), to first order in $\epsilon$ in the non-extremal Reissner-Nordstrom background, we find

$$
\partial_{r}\left[\left(r-r_{+}\right)\left(r-r_{-}\right) \partial_{r} \phi_{1}\right]=\frac{\beta^{2} \phi_{1}}{r^{2}}+\mathcal{O}(\varepsilon)
$$

where $\beta^{2}=\left.\frac{1}{2} \partial_{\phi}^{2} V_{\text {eff }}(\phi)\right|_{\phi=\phi_{0}}=\alpha^{2}|Q P|$.

It is convenient to define a new variable, $x$, given by

$$
x=\frac{r_{+}\left(r-r_{-}\right)+r_{-}\left(r-r_{+}\right)}{r\left(r_{+}-r_{-}\right)}=\left(r_{+}-r_{-}\right) \frac{r_{+} e^{-c \tau}+r_{-} e^{c \tau}}{e^{c \tau}-e^{-c \tau}},
$$

for which the inner and outer horizons are at $x_{ \pm}= \pm 1$ and $r=\infty$ is at $x_{\infty}=$ $\left(r_{+}+r_{-}\right) /\left(r_{+}-r_{-}\right)$.

In terms of $x$, the equation for $\phi_{1}$ is just Legendre's equation

$$
\partial_{x}\left(\left(x^{2}-1\right) \partial_{x} \phi_{1}\right)=\frac{1}{2} \alpha^{2} \phi_{1}
$$

which has the solution

$$
\phi_{1}=c_{1} P_{\gamma}(x)
$$

where $\gamma=\frac{1}{2}\left(\sqrt{1+2 \alpha^{2}}-1\right)$ and $P_{\gamma}$ is a Legendre function of the first kind. ${ }^{16}$ When $\gamma$ is an integer, $P_{\gamma}$ is a polynomial. It is amusing notice that this is the same $\gamma$ we used to categorise the exact solutions. Perhaps in these cases the perturbations series can be exactly summed.

To first order in $\varepsilon$, we find

$$
\phi(x)=\phi_{0}+\left(\phi_{\infty}-\phi_{0}\right) \frac{P_{\gamma}(x)}{P_{\gamma}\left(x_{\infty}\right)}
$$

In particular, deviation of the scalar field at the horizon from the attractor is just proportional to its deviation at infinity. Now, $x_{\infty}$ is inversely proportional to the temperature, so when the temperature is small, $x_{\infty}$ is large. Using the asymptotic form of the Legendre function [38], $P_{\gamma}\left(x_{\infty}\right) \sim x_{\infty}^{\gamma} \sim T^{-\gamma}$, we find that the deviation of the scalar field from the attractor value at the horizon goes like the deviation at infinity times the temperature to some power

$$
\left(\phi_{\text {Horizon }}-\phi_{\text {attractor }}\right) \sim\left(\phi_{\infty}-\phi_{\text {attractor }}\right) T^{\gamma}
$$

This formula holds for small deviations from the attractor values and small temperatures. Notice that in the limit $T \rightarrow 0$ we recover the attractor behaviour and for near extremal black holes we have approximate attractor behaviour as expected.

\footnotetext{
16 The other linearly independent solution is a Legendre function of the second kind which diverges at the horizon.
} 
Now we return to (4.38) and (4.39). Since the change to the metric are second order in $\varepsilon$, so are the changes of the coefficients $f_{i}$. We see that $V_{\text {eff }}$ remains unchanged to first order but, due to the square root on the right hand side of (4.39), $\partial_{\phi} V_{\text {eff }}$ changes to first order. So, to first order the scalar is governed by the same effective potential but it does not sit at the minimum at the horizon. In other words, for the non-extremal black hole, the infinite $A d S$ throat has been capped off and the scalar field does not have "time" to reach the minimum of its potential before it hits the horizon.

Although the scalar field is shifted, it does not necessarily follow that the entropy changes. Since changes to the metric appear at second order, the entropy actually remains unchanged to first order. However, we expect moduli dependence of the entropy to appear at second order. Since, in general, the second order perturbation analysis is rather complicated, we examined some of the exact solutions and found that moduli dependent corrections to the entropy do indeed appear at second order.

\section{6 (Un)attractors and special geometry}

The Lagrangian in (2) can be embedded in $\mathcal{N}=2$ supergravity theory for certain special values of the couplings. In this section, we briefly review the analysis of the (un)attractor equations in $\mathcal{N}=2$ special geometry language [17,29] and show the relation with our results.

The bosonic part of the $\mathcal{N}=2$ supergravity action coupled to arbitrary number of vector multiplets is given by ${ }^{17}$

$$
-\frac{R}{2}+G_{a \bar{a}} \partial_{\mu} z^{a} \partial_{\nu} \bar{z}^{\bar{a}}+\operatorname{Im}_{\Lambda \Sigma} \mathcal{N}_{\Lambda \Sigma} \mathcal{F}_{\mu \nu}^{\Lambda} \mathcal{F}_{\lambda \rho}^{\Sigma} g^{\mu \lambda} g^{\nu \rho}+\operatorname{Re}_{\Lambda \Sigma} \mathcal{F}_{\mu \nu}^{\Lambda} \star \mathcal{F}_{\lambda \rho}^{\Sigma} g^{\mu \lambda} g^{\nu \rho}
$$

Here $G_{a \bar{a}}$ is the metric of the scalar manifold and $\operatorname{Re\mathcal {N}}$ and $\operatorname{Im\mathcal {N}}$ components of $\mathcal{N}$ are negative definite scalar dependent vector couplings. Their explicit expressions can be obtained in terms of the symplectic sections of the underlying $\mathcal{N}=2$ theory and from the prepotential. The negative of the real and imaginary part of the vector couplings in the above $\mathcal{N}=2$ action can be schematically identified with our previous quantities $\tilde{f}_{a b}$ and $f_{a b}$, respectively.

One can construct the symplectic invariant quantity $\left.Z(z, \bar{z}, p, q)\right|^{2}+$ $\left|D_{a} Z(z, \bar{z}, p, q)\right|^{2}$ which can be identified with the scalar dependent effective potential $V_{\text {eff }}$. Here, $Z$ is the central charge in $\mathcal{N}=2$ supergravity theory and $D_{a} Z$ is the Kähler covariant derivative, $z$ are the complex moduli, $p$ and $q$ are the magnetic and electric charges, respectively. The central charge is given by the expression

$$
Z(z, \bar{z}, q, p)=e^{\frac{K(z, \bar{z}}{2}}\left(X^{\Lambda}(z) q_{\Lambda}-F_{\Lambda} p^{\Lambda}\right)
$$

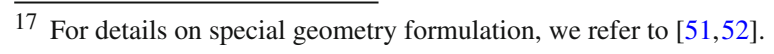


where $K(z, \bar{z})$ is the Kähler potential. So the effective potential is given in a simple form:

$$
V_{\mathrm{eff}}(z, \bar{z}, q, p)=|Z(z, \bar{z}, p, q)|^{2}+\left|D_{a} Z(z, \bar{z}, p, q)\right|^{2}
$$

The above form of $V_{\text {eff }}$ can be simplified to obtain an expression in terms of electric, magnetic charges as well as the real and imaginary part of the vector coupling $\mathcal{N}$ :

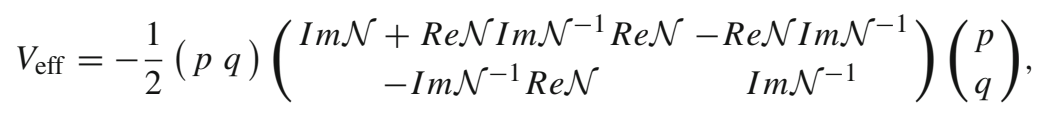

where, we have suppressed the indices $I, J$. This is equivalent to the expression for the effective potential obtained in [25] which has been derived by using the metric ansatz and the equations of motion.

The metric of the spherically symmetric solution is given by

$$
d s^{2}=e^{2 U} d t^{2}-e^{-2 U}\left[\frac{c^{4}}{\sinh ^{4} c \tau} d \tau^{2}+\frac{c^{2}}{\sinh ^{2} c \tau} d \Omega^{2}\right]
$$

Then the constraint becomes,

$$
\left(\frac{d U}{d \tau}\right)^{2}+\left|\frac{d z}{d \tau}\right|^{2}+e^{2 U}\left(|Z(z, \bar{z}, q, p)|^{2}+\left|D_{a} Z(z, \bar{z}, q, p)\right|^{2}\right)=c^{2}
$$

The constraint expression evaluated at infinity (at $\tau \rightarrow 0, U \rightarrow M \tau$ ) is given by,

$$
\begin{aligned}
& M^{2}\left(z_{\infty}, \bar{z}_{\infty}, p, q\right)-\left|Z\left(z_{\infty}, \bar{z}_{\infty}, p, q\right)\right|^{2} \\
& \quad=c^{2}+\left|D_{a} Z\left(z_{\infty}, \bar{z}_{\infty}, p, q\right)\right|^{2}-G_{a \bar{a}} \Sigma^{a} \bar{\Sigma}^{\bar{a}}
\end{aligned}
$$

For BPS configuration,

$$
\begin{aligned}
M^{2}\left(z_{\infty} \bar{z}_{\infty}, p, q\right) & =\left|Z\left(z_{\infty}, \bar{z}_{\infty}, p, q\right)\right|^{2}, \\
c & =0, \quad G^{a \bar{a}} \bar{D}_{\bar{a}} Z\left(z_{\infty}, \bar{z}_{\infty}, p, q\right)=\Sigma^{a}
\end{aligned}
$$

For extremal solution, $c^{2}=2 S T=0$, (here we have used a different normalization for the parameter $c$ as compared to our previous discussion) but when $D_{a} Z(z, \bar{z}, p, q) \neq 0$, this describes non BPS solutions. For non-extremal solutions $c^{2} \neq 0$ and $D_{a} Z(z, \bar{z}, p, q) \neq 0$.

The condition for the attractor is obtained by knowing the critical point of the effective potential. Using special geometry identities, the critical point of $V_{\text {eff }}$ is given by the expression,

$$
\partial_{a} V_{\text {eff }}=2\left(D_{a} Z\right) \bar{Z}+i C_{a b c} G^{b \bar{m}} G^{c \bar{n}} \bar{D}_{\bar{m}} \bar{Z} \bar{D}_{\bar{n}} \bar{Z}
$$


This shows that l.h.s. is zero when $D_{a} Z=\bar{D}_{\bar{a}} \bar{Z}=0$ which means that the critical point of $V_{\text {eff }}$ coincides with the critical point of the central charge.

The second condition for the existence of an attractor is obtained by evaluating the second derivative of the effective potential at the critical point. Using special geometry identities, one gets,

$$
\left(\bar{D}_{\bar{a}} D_{b} V_{\mathrm{eff}}\right)_{c r}=\left(\bar{\partial}_{\bar{a}} \partial_{b} V_{\mathrm{eff}}\right)_{c r}=2 G_{\bar{a} b} V_{\mathrm{eff}}(c r)
$$

which shows that the sign of the second derivative of the effective potential is positive when the sign of the moduli space metric at the critical point is positive.

Equivalently, one can also obtain these conditions by considering the equation of motion for the scalars and assuming the moduli space to be a complex Kähler manifold with a Kähler metric $G_{a \bar{b}}$.

The equation of motion for the scalar is given by [29],

$$
\partial_{\tau}\left(\partial_{\tau} z^{a}\right)+\Gamma_{b c}^{a}(z, \bar{z}) \partial_{\tau} z^{b} \partial_{\tau} z^{c}=G^{a \bar{b}} e^{2 U} \frac{\partial V}{\partial \bar{z}^{\bar{b}}}
$$

For extremal (BPS and non BPS) solution, the 1.h.s. at the horizon is zero, so one gets the condition

$$
\left.\frac{\partial V}{\partial z^{a}}\right|_{z_{h}^{a}}=0
$$

where, $z_{h}^{a}$ is the value of the scalar field at the horizon.

For non-extremal case, the attractor equation reduces to (after some change of coordinates),

$$
\left.\left(z^{a}\right)^{\prime \prime}\right|_{\rho=0}=\left.g^{a \bar{b}} \frac{\partial V}{\partial \bar{z}^{\bar{b}}}\right|_{z_{h}^{a}} .
$$

Here, $\rho \rightarrow 0$ is the near horizon limit and $\rho$ is related to $\tau$ as $\rho=2 e^{c \tau}$. The metric function in the new coordinate is given as, $e^{2 U} \rightarrow\left(-c^{2} \rho^{2} / r_{h}^{2}\right)$. The near horizon geometry is then given as:

$$
d s^{2}=\rho^{2} d t^{2}-\left(r_{h}\right)^{2} d \rho^{2}-\left(r_{h}\right)^{2} d \Omega^{2}, \quad \rho \rightarrow 0
$$

Certainly, the l.h.s. of the attractor equation (133), is not zero and hence the derivative of the effective potential is not zero and hence there is no attractor phenomenon. This is reflected in our equation (108) which has been derived from the scalar field equation of motion and using the horizon values of the derivative of the moduli as well as $a^{2}$, $b^{2}$ appearing in the metric function. We have shown there that the r.h.s. of Eq. (108) is not equal to zero. This shows that the attractor phenomenon does not occur in the non-extremal case. 


\section{The role of non-supersymmetric attractor in microscopic/macroscopic entropy matching}

The extremality condition was enough to constrain the near-horizon geometry and ensure that the entropy is independent of the asymptotic values of the moduli-the entropy only depends on the modulus independent product $\bar{P} \bar{Q}=P Q$. In this section $^{18}$ we argue that the attractor mechanism is at the basis of the matching between microscopic and macroscopic entropy of certain extremal non-BPS black holes. In particular, we consider the examples as discussed in [15,30].

The thermodynamics of extremal black holes is very tricky (see, also, the Sect. 6). In many cases, the horizon area is finite and one expects the entropy to be non-zero. Therefore, a vanishing entropy on the Euclidean section should prevent us from trusting the Euclidean semi-classical calculations. A strong argument to support a nonvanishing entropy for extremal black holes comes from string theory which provides a nice microscopic interpretation. In string theory, the entropy of an extremal BPS black hole is computed by counting the degeneracy of D-branes states-D-branes are the constituents from which the black hole is formed. That is equivalent to counting the BPS states (lowest mass states at fixed charges) in the D-brane world-volume theory. Supersymmetry is at the basis of the non-renormalization theorems that ensures that the ground state degeneracy is a kinematic quantity rather than a dynamical one (it is independent of the strength of the string coupling). Then, the counting of the number of D-branes at weak coupling agrees with the classical area law of the black hole at strong coupling.

The large ${ }^{19}$ non-susy black holes share an important property with their BPS cousins: they have the lowest possible mass in the quantum theory (due to the extremality condition) and there is no other black hole state to which they can decay by Hawking radiation. Then, their temperature should vanish. The extremality condition acts as the cosmic censorship preventing a minimum mass black hole to decay in a naked singularity. An important question arises here, namely, is there any D-brane microscopic configuration to describe such a non-BPS black hole? The answer is affirmative and in what follows, we present a concrete example.

In [15], an intriguing example of microstates counting for a neutral black hole has been proposed that precisely reproduces the Hawking-Bekenstein entropy. The nonrotating case corresponds to our solution II. This solution can be embedded in string theory [32] by identifying the KK circle with the M-theory circle. In this way, the magnetic charge becomes the charge of the D- 6 brane and the electric charge is the D-0 brane charge.

We will discuss this case using the effective potential formalism, but the computations using the entropy function are very similar with the calculations in the Sect. 4.2. The effective potential is given by

$$
V_{\mathrm{eff}}=e^{\alpha \phi}(Q)^{2}+e^{-\alpha \phi}(P)^{2},
$$

\footnotetext{
18 DA would like to thank Ashoke Sen for discussions on this section.

19 Large black holes are the black holes for which the spacetime curvature is weak outside the horizon-of course when the curvature is strong, e.g. is blowing up at the singularity, the stringy effects are important.
} 
with $\alpha=2 \sqrt{3}$. Then, the value of the modulus at the horizon and the entropy are

$$
e^{4 \sqrt{3} \phi_{H}}=\frac{P^{2}}{Q^{2}}, \quad S=\pi V_{\mathrm{eff}}\left(\phi_{H}\right)=2 \pi P Q .
$$

These expressions involve only the charge parameters, the moduli cancel out. This is interpreted as a signal that a clear connection to the microscopic theory is possible. This black hole is not supersymmetric and this is in agreement with the absence of the bound states of $D_{0}$ and $D_{6}$ branes. However, in [15], a simple string description based on nonsupersymmetric, quadratically stable, $D_{0}-D_{6}$ bound states [49] was provided - this statistical prescription precisely reproduces the Hawking-Bekenstein entropy.

The electric and magnetic charges are quantized according to Dirac's quantization rule and we obtain

$$
\frac{2 P Q}{G_{4}}=n_{Q} n_{P},
$$

where the integers $n_{Q}$ and $n_{P}$ can be interpreted after the embedding in M-theory as the number of $D 0$ - and $D 6$-branes, respectively. The entropy of extremal black hole can be rewritten [32] as

$$
S=\pi n_{P} n_{Q}=\pi N_{0} N_{6}
$$

The excitations of the D-branes system at low energies are described in terms of a moduli space approximation. Since our black hole is non-BPS, one can not use the non-renormalization theorems to argue that low energy theory does not receive corrections when the string coupling is increased. Therefore, the black hole dynamics in the strong coupling regime is described by a different moduli space. Now, it is important to remember that the near-horizon geometry of the non-BPS extremal black holes is the same as for the BPS extremal black holes, namely $A d S_{2} \times S^{2}$. Then even if we start with different moduli spaces (in different regimes), the moduli are attracted to the horizon to the same values. Then, this seems to be the reason for the mysterious microscopic/macroscopic entropy match in [15].

We will comment more on the validity of our proposal in Sect. 6.

\section{Discussion}

In classical general relativity, no hair theorems impose strong constraints on the possibility of obtaining solutions of the Einstein equations coupled to non-trivial scalar fields. A crucial ingredient for their proof is that the scalars be minimally coupled to gravity and other fields. In this paper, we have considered black holes with scalar fields which are non-minimally coupled to gauge fields. Clearly, this is a concrete possibility for evading no hair theorems. Indeed, we have seen how the non-zero asymptotic scalar charges and the values of moduli at infinity play a role in the first law of thermodynamics. However, this is not considered as a drastic violation of the no hair theorems $[8,9,46]$. The reason is that the scalar charges are not independent parameters, but are given functions of the other asymptotic charges which characterise the solution. 
Let us consider, for example, the part of the Lagrangian containing the moduli and the moduli coupled to gauge fields. The Lagrangian reduces to the standard EinsteinMaxwell form if the moduli are constant. However if $F_{\mu \nu} F^{\mu \nu} \neq 0$ and the scalar field is not at the minimum of its effective potential, the field equation for $\phi$ will not be satisfied by taking $\phi$ to be a constant.

This means that the non-vanishing electromagnetic field can also be understood as a source for the moduli. As a result, the scalar charges have been called secondary hair by the authors of $[8,9,46] .{ }^{20}$ They are generated because the basic fields (associated with mass, angular momentum, and gauge charges) act also as sources for the moduli. This should be contrasted with primary hair which would be due an asymptotic scalar charge which is completely independent of the other charges. It is significant that they do not represent a new quantum number associated with the black hole. However, in string theory the scalar fields, referred to as moduli are interpreted as local coupling constants rather than matter fields and the notion of scalar charge is somehow misleading. While conventional in general relativity, one would not normally consider variations of the moduli at infinity, there are case in both string theory and general relativity where one might be lead to consider them hence introducing a new term in the first law (59). For example, one may be interested in time-dependent cosmological situations in which $\phi_{\infty}$ becomes dynamical, ${ }^{21}$ one may wish to understand the behaviour of the black holes under slow adiabatic changes of $\phi_{\infty}$ or one may wish to compare black holes at different points in moduli space.

The non-extremal black holes have a non-zero temperature that can be evaluated by eliminating the conical singularity in the Euclidean section. Then, the Euclidean geometry becomes a "cigar" and so the Euclidean time circle closes off smoothly. On the other hand, for an extremal Euclidean black hole the topology changes. The Euclidean time circle does not close off and so there is no conical singularity. In this case, one is forced, either to work with an arbitrary periodicity of the Euclidean time leading to ambiguous results, or simply to ignore the Euclidean time method. However, in the Lorentzian section the picture is quite satisfactory: an extremal black hole is obtained by continuously sending the surface gravity of a non-extremal black hole to zero. While the surface gravity (i.e. the temperature) vanishes, the area of the horizon (i.e. the entropy) can remain finite. These results strongly suggest that the entropy of an extremal black hole with a non-vanishing horizon area is non-zero.

The extremal supersymmetric black holes play a central role in providing a statistical foundation for black hole thermodynamics in string theory. In all known cases, supersymmetric (static) black holes are also extreme black holes-the converse is not true. This can be understood by the fact that the BPS black holes are stable systems corresponding to the lowest possible mass in the quantum theory and should not radiate. Then, their temperature should vanish and so they are extremal. On the other hand, not all extremal black holes saturate BPS bounds and they can break supersymmetry-the

\footnotetext{
20 We prefer the term stubble.

21 Since the derivation assumes asymptotically flat space, one would require sufficiently flat background for the analysis to remain valid.
} 
mismatch between the extremality and BPS conditions is quite general [39]. There are two kinds of extremal non-susy solutions. The first one contains extremal black holes which can not be embedded in supergravity theories (e.g., a subset of the solutions Ib). The second one contains extremal black holes which are solutions of supergravity theories but are not supersymmetric (e.g., the solution II).

It was discovered long time ago that, in four-dimensional, ungauged $\mathcal{N}=2$ supergravity, the BPS black hole solutions exhibit fixed-point attractor behavior near the horizon. However, recently it was understood that the near-horizon extremal geometry [44] is at the basis of the attractor mechanism, rather than supersymmetry. It is just more convenient to solve the supersymmetry transformations for the gravitino and gauginos in a bosonic background of $(\mathcal{N}=2)$ supergravity-these transformations depend linearly on the first derivatives. Consequently, to find BPS black hole solutions one has to solve first order differential equations (the attractor equations near-horizon become algebraic) [40,41].

When the BPS bound is saturated, the entropy is determined microscopically just by the charges. However, the charges are quantized and then the entropy should also be a discrete quantity. Instead, the moduli are continuous parameters of the internal manifold. For consistency with the discreteness of the entropy, the values of the moduli at the horizon can not have any arbitrary values. The attractor mechanism provide an explanation for why the moduli are fixed at the horizon. However, this argument is not based at all on supersymmetry and this was one important reason for investigating non-supersymmetric attractors in [25].

In the previous section, we proposed that the attractor mechanism is at the origin of the microscopic/macroscopic match of some non-BPS extremal black holes $[15,30]$ (see, also, [10] for a five-dimensional example). We used the effective potential method to show that the Kaluza-Klein horizon is an attractor for the moduli and have explicitly shown that there is just one minimum. This is the explanation for why the entropy is independent of continuous parameters (coupling constants). It is also worth to be mentioned that the existence of just one minimum imply that there can not be jumps in entropy moving from weak coupling to strong coupling coupling. Although this proposal is certainly suggestive, one should take it with some caution-similar arguments are not valid when the basin of attraction is not unique. In general, the effective potential method will provide more information about the attractor behavior than the entropy function. For example, if there is more than one attractor fixed point, then a study of the effective potential will make it clear which minimum can be obtained by starting with different boundary conditions. In this case, our arguments fail, because by changing the coupling $g_{s} Q$, the moduli can end up in a different domain of attraction and the value of the entropy will change. ${ }^{22}$ For the KK solution we have found that the effective potential has just one minimum and we expect this is also true for the black holes of [30]. Another point worth to be mentioned is that, for consistency with the macroscopic picture, the microscopic configuration of branes should be also non-supersymmetric but stable. For the case at hand-KK black hole-it is known [49] that, indeed, this is true. The 0-brane and the 6-brane repel one another and so, in

22 The near-horizon geometry remains $A d S_{2} \times S^{2}$ even after adding $\alpha^{\prime}$ corrections-the radia of $A d S_{2}$ and $S^{2}$ receive corrections, but the geometry does not change. 
general, a point-like 0-brane placed on or near a 6-brane gives rise to a non-susy configuration. However, a D0-D6 brane configuration has been proposed in [49] which satisfies the classical equations of motion and is classically stable to quadratic order. That is, a set of four 0-branes which are smeared out over four 6-branes wrapped on a six-torus - this configuration served as a basis of the microscopic picture in [15]. These metastable states are interpreted as some kind of long-lived resonances composed of 0-branes and 6-branes and so the microscopic and the macroscopic pictures are consistent with one another. One more puzzle is related to the lack of non-renormalization theorems for the extremal non-BPS black holes. In the strong coupling regime the extremal black hole can still be thought of as the black hole with the lowest mass. However, by changing the coupling, the mass will receive corrections and the statistical entropy definition should be revised.

The counting in [15] requires $N_{6} \gg 4$, but the configurations constructed in [49] can be found even for small numbers of branes. It will also be interesting to reproduce the entropy of KK black hole in this case. Our investigation in Sect. 4.4 strongly suggests that, due to the attractor mechanism, a computation of the near-extremal KK black hole entropy with large charges should be also possible.

The rotating case was also studied in [2] by using the entropy function. It is worth noticing that the long throat of $A d S_{2}$ is also present in the near-horizon geometry of the extremal rotating black hole. Unfortunately, in the rotating case, it is difficult to construct an effective potential when the moduli are not constants. It will be interesting to find an effective potential analogous to (107) and study the near-extremal rotating black holes. It will also be interesting to investigate the thermodynamics of the non-extremal black holes by using the "counter-term" method developed in $[3,4,36]$.

Acknowledgments The authors would like to thank Ashoke Sen for suggestions, useful discussions, and sharing with them some of his related ideas. We would also like to thank Sandip Trivedi for interesting conversations. DA would like to thank the members of HRI string group for their feedback during the discussions at String lunch. The work of D.A. and K.G. is supported by the Department of Atomic Energy, Government of India.

Open Access This article is distributed under the terms of the Creative Commons Attribution Noncommercial License which permits any noncommercial use, distribution, and reproduction in any medium, provided the original author(s) and source are credited.

\section{Appendix A: Effective potential}

Let us derive the expression of the effective potential, $V_{\text {eff }}$, by rewriting (4) as (11). We have

$$
\begin{aligned}
& \partial_{\mu}\left(\sqrt{-G} g_{i j} G^{\mu \nu} \partial_{\nu} \phi^{j}\right) \\
& =\sqrt{-G}\left[\frac{1}{4} \frac{\partial f_{A B}}{\partial \phi^{i}} F_{\mu \nu}^{A} F^{B \mu \nu}+\frac{1}{8} \frac{\partial \tilde{f}_{A B}}{\partial \phi^{i}} F_{\mu \nu}^{A} F_{\rho \sigma}^{B} \epsilon^{\mu \nu \rho \sigma}\right],
\end{aligned}
$$


with $\sqrt{-G}=b^{2} \sin \theta$. Then

$$
\begin{aligned}
& \partial_{r}\left(b^{2} \sin \theta g_{i j} G^{r r} \partial_{r} \phi^{j}\right) \\
& \quad=\left(b^{2} \sin \theta\right)\left[\frac{1}{4} \frac{\partial f_{A B}}{\partial \phi^{i}} F_{\mu \nu}^{A} F^{B \mu \nu}+\frac{1}{8} \frac{\partial \tilde{f}_{A B}}{\partial \phi^{i}} F_{\mu \nu}^{A} F_{\rho \sigma}^{B} \epsilon^{\mu \nu \rho \sigma}\right],
\end{aligned}
$$

and

$$
\partial_{r}\left(b^{2} a^{2} g_{i j} \partial_{r} \phi^{j}\right)=b^{2}\left[\frac{1}{4} \frac{\partial f_{A B}}{\partial \phi^{i}} F_{\mu \nu}^{A} F^{B \mu \nu}+\frac{1}{8} \frac{\partial \tilde{f}_{A B}}{\partial \phi^{i}} F_{\mu \nu}^{A} F_{\rho \sigma}^{B} \epsilon^{\mu \nu \rho \sigma}\right] .
$$

Now, we calculate $V_{\text {eff }}$ by using its definition (11):

$$
\frac{\partial V_{\mathrm{eff}}}{\partial \phi^{i}}=2 b^{4}\left[\frac{1}{4} \frac{\partial f_{A B}}{\partial \phi^{i}} F_{\mu \nu}^{A} F^{B \mu \nu}+\frac{1}{8} \frac{\partial \tilde{f}_{A B}}{\partial \phi^{i}} F_{\mu \nu}^{A} F_{\rho \sigma}^{B} \epsilon^{\mu \nu \rho \sigma}\right]
$$

To get (12), we need to use (7)

$$
\begin{aligned}
F^{A} & =f^{A B}\left(Q_{B}-\tilde{f}_{B C} P^{C}\right) \frac{1}{b^{2}} d t \wedge d r+P^{A} \sin \theta d \theta \wedge d \phi \\
& =F_{t r}^{A} d t \wedge d r+F_{\theta \phi}^{A} d \theta \wedge d \phi
\end{aligned}
$$

and so we find

$$
\begin{aligned}
\frac{1}{4} & \frac{\partial f_{A B}}{\partial \phi^{i}} F_{\mu \nu}^{A} F^{B \mu \nu} \\
& =\frac{1}{4} 2 \frac{\partial f_{A B}}{\partial \phi^{i}}\left(G^{r r} G^{t t} F_{t r}^{A} F_{t r}^{B}+G^{\theta \theta} G^{\phi \phi} F_{\theta \phi}^{A} F_{\theta \phi}^{B}\right) \\
& =\frac{1}{2}\left[-\frac{\partial f_{A B}}{\partial \phi^{i}} f^{A C}\left(Q_{C}-\tilde{f}_{C D} P^{D}\right) \frac{1}{b^{2}} f^{B E}\left(Q_{E}-\tilde{f}_{E F} P^{F}\right) \frac{1}{b^{2}}+\frac{\partial f_{A B}}{\partial \phi^{i}} \frac{1}{b^{4}} P^{A} P^{B}\right], \\
& =\frac{1}{2}\left[\frac{\partial f^{A C}}{\partial \phi^{i}} f_{A B}\left(Q_{C}-\tilde{f}_{C D} P^{D}\right) \frac{1}{b^{2}} f^{B E}\left(Q_{E}-\tilde{f}_{E F} P^{F}\right) \frac{1}{b^{2}}+\frac{\partial f_{A B}}{\partial \phi^{i}} \frac{1}{b^{4}} P^{A} P^{B}\right], \\
& =\frac{1}{2}\left[\frac{\partial f^{A C}}{\partial \phi^{i}}\left(Q_{C}-\tilde{f}_{C D} P^{D}\right) \frac{1}{b^{2}}\left(Q_{A}-\tilde{f}_{A F} P^{F}\right) \frac{1}{b^{2}}+\frac{\partial f_{A B}}{\partial \phi^{i}} \frac{1}{b^{4}} P^{A} P^{B}\right],
\end{aligned}
$$

and also $\left(F_{r t}=-F_{t r}\right)$

$\frac{1}{8} \frac{\partial \tilde{f}_{A B}}{\partial \phi^{i}} F_{\mu \nu}^{A} F_{\rho \sigma}^{B} \epsilon^{\mu \nu \rho \sigma}=\frac{\partial \tilde{f}_{A B}}{\partial \phi^{i}} F_{r t}^{B} F_{\theta \phi}^{A}=-\frac{\partial \tilde{f}_{A B}}{\partial \phi^{i}} f^{B E}\left(Q_{E}-\tilde{f}_{E F} P^{F}\right) \frac{1}{b^{2}} \frac{P^{A}}{b^{2}}$ 
Then, using $f_{A B} \partial f^{A B}=-f^{A B} \partial f_{A B},(142)$ becomes

$$
\begin{aligned}
\frac{\partial V_{\mathrm{eff}}}{\partial \phi^{i}}= & {\left[\frac{\partial f^{A C}}{\partial \phi^{i}}\left(Q_{C}-\tilde{f}_{C D} P^{D}\right)\left(Q_{A}-\tilde{f}_{A F} P^{F}\right)+\frac{\partial f_{A B}}{\partial \phi^{i}} P^{A} P^{B}\right] } \\
& -2 \frac{\partial \tilde{f}_{A B}}{\partial \phi^{i}} f^{B E}\left(Q_{E}-\tilde{f}_{E F} P^{F}\right) P^{A}
\end{aligned}
$$

One can easily check that the effective potential (12) satisfies the previous equation.

\section{Appendix B: Toda equations}

We rewrite the Eqs. (24)-(25) in a form similar to Toda equations. We define

$$
A=2 u_{2}+\alpha_{1} u_{1}, \quad B=2 u_{2}+\alpha_{2} u_{1},
$$

and we obtain the following equivalent system

$$
\begin{aligned}
& \ddot{A}=\left(\frac{1}{2} \alpha_{1}^{2}+2\right) Q_{1}^{2} e^{A}+\left(\frac{1}{2} \alpha_{1} \alpha_{2}+2\right) Q_{2}^{2} e^{B}, \\
& \ddot{B}=\left(\frac{1}{2} \alpha_{1} \alpha_{2}+2\right) Q_{1}^{2} e^{A}+\left(\frac{1}{2} \alpha_{2}^{2}+2\right) Q_{2}^{2} e^{B} .
\end{aligned}
$$

For $\alpha_{1} \alpha_{2}=-4$ the equations decouple and we obtain

$$
\begin{aligned}
& \ddot{A}=\left(\frac{1}{2} \alpha_{1}^{2}+2\right) Q_{1}^{2} e^{A}=e^{(A+a)}=e^{\bar{A}}, \\
& \ddot{B}=\left(\frac{1}{2} \alpha_{2}^{2}+2\right) Q_{2}^{2} e^{B}=e^{(B+b)}=e^{\bar{B}} .
\end{aligned}
$$

A solution of the equation $\ddot{X}=e^{X}$ can be written in the following form:

$$
X=\log \left(\frac{2 c^{2}}{\sinh ^{2}(c(\tau-d))}\right)=\log \left(\frac{2 c^{2}}{F^{2}(\tau)}\right) .
$$

The solutions are given by

$$
\begin{aligned}
& \bar{A}=A+a=\log \left(\frac{2 c_{1}^{2}}{F_{1}^{2}(\tau)}\right) \Rightarrow A=\log \left(\frac{2 c_{1}^{2}}{F_{1}^{2}(\tau)} \frac{2}{Q_{1}^{2}\left(\alpha_{1}^{2}+4\right)}\right) \\
& \bar{B}=B+b=\log \left(\frac{2 c_{2}^{2}}{F_{2}^{2}(\tau)}\right) \Rightarrow A=\log \left(\frac{2 c_{2}^{2}}{F_{2}^{2}(\tau)} \frac{2}{Q_{2}^{2}\left(\alpha_{2}^{2}+4\right)}\right)
\end{aligned}
$$




\section{Appendix C: Finding solutions}

We construct the solution I with $\gamma=1 \Leftrightarrow \alpha_{1} \alpha_{2}=-4$. Without loss of generality we assume $\alpha_{2}<0$. We obtain the following expression for the dilaton, $a^{2}$, and $\tau$ (we define $\left.\lambda=\frac{\alpha_{1}}{\alpha_{1}-\alpha_{2}}\right)$ :

$$
\begin{aligned}
e^{\left(\alpha_{1}-\alpha_{2}\right) \phi} & =e^{A-B}=\left(\frac{F_{2} c_{1}}{F_{1} c_{2}}\right)^{2} e^{(b-a)}=\left(\frac{F_{2} c_{1}}{F_{1} c_{2}}\right)^{2} \frac{Q_{2}^{2}\left(\alpha_{2}^{2}+4\right)}{Q_{1}^{2}\left(\alpha_{1}^{2}+4\right)} \\
a^{2} & =\frac{4}{\left(\alpha_{1}^{2}+4\right)^{1-\lambda}\left(\alpha_{2}^{2}+4\right)^{\lambda}}\left(\frac{F_{1} Q_{1}}{c_{1}}\right)^{2(\lambda-1)}\left(\frac{c_{2}}{F_{2} Q_{2}}\right)^{2 \lambda} \\
\tau & =\int \frac{d r}{a^{2} b^{2}}=\frac{1}{\left(r_{+}-r_{-}\right)} \log \left(\frac{r-r_{+}}{r-r_{-}}\right)
\end{aligned}
$$

Appendix C.1: Boundary conditions

- Horizon $\left(r \rightarrow r_{+}, \tau \rightarrow-\infty\right)$

As $r \rightarrow r_{+}($ie. $\tau \rightarrow-\infty)$ the scalar field goes like

$$
e^{\left(\alpha_{1}-\alpha_{2}\right) \phi} \sim e^{2\left(c_{1}-c_{2}\right) \tau}
$$

so, for $\phi$ finite at the horizon, we require $c:=c_{1}=c_{2}$. Also at the horizon

$$
b^{2} \sim\left(r-r_{+}\right) / a^{2} \sim\left(r-r_{+}\right)\left(\frac{r-r_{-}}{r-r_{+}}\right)^{\frac{2 c}{r_{+}-r_{-}}}
$$

which necessitates

$$
\left(r_{+}-r_{-}\right)=2 c
$$

- Asymptotic infinity $(r \rightarrow \infty, \tau \rightarrow 0)$

At infinity, the scalar field tends to

$$
e^{\left(\alpha_{1}-\alpha_{2}\right) \phi_{\infty}}=e^{\left(\alpha_{1}-\alpha_{2}\right) \phi(\tau=0)}=\frac{\alpha_{2}^{2}+4}{\alpha_{1}^{2}+4}\left(\frac{Q_{2} \sinh \left(c d_{2}\right)}{Q_{1} \sinh \left(c d_{1}\right)}\right)^{2}
$$

which we can write

$$
\left(\alpha_{1}^{2}+4\right) \bar{Q}_{1}^{2} \sinh ^{2}\left(c d_{1}\right)=\left(\alpha_{2}^{2}+4\right) \bar{Q}_{2}^{2} \sinh ^{2}\left(c d_{2}\right)
$$

where

$$
\bar{Q}_{i}^{2}=e^{\alpha_{i} \phi_{\infty}} Q_{i}^{2}
$$


We also have

$$
\begin{aligned}
\left.a^{2}\right|_{\tau=0}=1 & =\frac{4}{\left(\alpha_{1}^{2}+4\right)^{1-\lambda}\left(\alpha_{2}^{2}+4\right)^{\lambda}} c^{2}\left(Q_{1} \sinh \left(c d_{1}\right)\right)^{-2(1-\lambda)}\left(Q_{2} \sinh \left(c d_{2}\right)\right)^{-2 \lambda} \\
& =\frac{4}{\left(\alpha_{1}^{2}+4\right)^{1-\lambda}\left(\alpha_{2}^{2}+4\right)^{\lambda}} c^{2}\left(\bar{Q}_{1}^{2} \sinh ^{2}\left(c d_{1}\right)\right)^{-(1-\lambda)}\left(\bar{Q}_{2}^{2} \sinh ^{2}\left(c d_{2}\right)\right)^{-\lambda}
\end{aligned}
$$

Together with (164) this implies

$$
\begin{aligned}
& \sinh \left(c d_{1}\right)=2 c\left(\alpha_{1}^{2}+4\right)^{-\frac{1}{2}} \bar{Q}_{1}^{-1} \\
& \sinh \left(c d_{2}\right)=2 c\left(\alpha_{2}^{2}+4\right)^{-\frac{1}{2}} \bar{Q}_{2}^{-1}
\end{aligned}
$$

The scalar "charge" is

$$
-\dot{\phi}(\tau=0)=\Sigma=-\frac{2 c\left(\operatorname{coth}\left(c d_{1}\right)-\operatorname{coth}\left(c d_{2}\right)\right)}{\alpha_{1}-\alpha_{2}}
$$

and the mass is

$$
\begin{aligned}
\left.\dot{a}\right|_{\tau=0}=M= & \overbrace{\left(\frac{2 c\left(Q_{1} \sinh \left(c d_{1}\right)\right)^{-(1-\lambda)}\left(Q_{2} \sinh \left(c d_{2}\right)\right)^{-\lambda}}{\left[\left(\alpha_{1}^{2}+4\right)^{1-\lambda}\left(\alpha_{2}^{2}+4\right)^{\lambda}\right]^{\frac{1}{2}}}\right)}^{1} c\left(\lambda \operatorname{coth}\left(c d_{2}\right)\right. \\
& \left.+(1-\lambda) \operatorname{coth}\left(c d_{1}\right)\right) \\
= & c\left(\lambda \operatorname{coth}\left(c d_{2}\right)+(1-\lambda) \operatorname{coth}\left(c d_{1}\right)\right) .
\end{aligned}
$$

The entropy is given by

$$
S=\pi b^{2}(\tau=-\infty)=\frac{\pi}{4}\left(\alpha_{1}^{2}+4\right)^{(1-\lambda)}\left(\alpha_{2}^{2}+4\right)^{\lambda}\left(Q_{1}^{2} e^{2 c d_{1}}\right)^{(1-\lambda)}\left(Q_{2}^{2} e^{2 c d_{2}}\right)^{\lambda}
$$

while the temperature is

$$
\begin{aligned}
T & =\frac{a a^{\prime}}{2 \pi}(\tau=-\infty) \\
& =\frac{c}{2 \pi} \frac{4}{\left(\alpha_{1}^{2}+4\right)^{(1-\lambda)}\left(\alpha_{2}^{2}+4\right)^{\lambda}}\left(Q_{1}^{2} e^{-2 c d_{1}}\right)^{-(1-\lambda)}\left(Q_{2}^{2} e^{-2 c d_{1}}\right)^{-\lambda}
\end{aligned}
$$

and as a check, we note that,

$$
S T=\frac{c}{2}=\frac{1}{4}\left(r_{+}-r_{-}\right),
$$


and one can also check that

$$
M^{2}+\Sigma^{2}-\bar{Q}_{1}^{2}-\bar{Q}_{2}^{2}=4 S^{2} T^{2}=c^{2}
$$

\section{References}

1. Alishahiha, M., Ebrahim, H.: Non-supersymmetric attractors and entropy function. JHEP 03, 003 (2006)

2. Astefanesei, D., Goldstein, K., Jena, R.P., Sen, A., Trivedi, S.P.: Rotating attractors. JHEP 10, 058 (2006)

3. Astefanesei, D., Mann, R.B., Stelea, C.: Note on counterterms in asymptotically flat spacetimes. Phys. Rev. D75, 024,007 (2007)

4. Astefanesei, D., Radu, E.: Quasilocal formalism and black ring thermodynamics. Phys. Rev. D73, 044,014 (2006)

5. Callan Curtis, G.J., Maldacena, J.M.: D-brane approach to black hole quantum mechanics. Nucl. Phys. B472, 591-610 (1996)

6. Cardoso, G.L., Grass, V., Lust, D., Perz, J.: Extremal non-bps black holes and entropy extremization. JHEP 09, 078 (2006)

7. Chandrasekhar, B., Parvizi, S., Tavanfar, A., Yavartanoo, H.: Non-supersymmetric attractors in $\mathrm{r}^{* * 2}$ gravities. JHEP 08, 004 (2006)

8. Coleman, S.R., Preskill, J., Wilczek, F.: Growing hair on black holes. Phys. Rev. Lett. 67, 1975-1978 (1991)

9. Coleman, S.R., Preskill, J., Wilczek, F.: Quantum hair on black holes. Nucl. Phys. B378, 175-246 (1992)

10. Dabholkar, A.: Microstates of non-supersymmetric black holes. Phys. Lett. B402, 53-58 (1997)

11. Dabholkar, A., Sen, A., Trivedi, S.P.: Black hole microstates and attractor without supersymmetry. JHEP 01, 096 (2007)

12. Denef, F.: Supergravity flows and d-brane stability. JHEP 08, 050 (2000)

13. Denef, F., Greene, B.R., Raugas, M.: Split attractor flows and the spectrum of bps d-branes on the quintic. JHEP 05, 012 (2001)

14. Dobiasch, P., Maison, D.: Stationary, spherically symmetric solutions of Jordan's unified theory of gravity and electromagnetism. Gen. Relat. Grav. 14, 231-242 (1982)

15. Emparan, R., Horowitz, G.T.: Microstates of a neutral black hole in $m$ theory. Phys. Rev. Lett. 97, 141,601 (2006)

16. Ferrara, S.: Bertotti-Robinson geometry and supersymmetry [hep-th/9701163] (1997)

17. Ferrara, S., Gibbons, G.W., Kallosh, R.: Black holes and critical points in moduli space. Nucl. Phys. B500, 75-93 (1997)

18. Ferrara, S., Gimon, E.G., Kallosh, R.: Magic supergravities, $n=8$ and black hole composites. Phys. Rev. D74, 125,018 (2006)

19. Ferrara, S., Kallosh, R.: Supersymmetry and attractors. Phys. Rev. D54, 1514-1524 (1996)

20. Ferrara, S., Kallosh, R.: Universality of supersymmetric attractors. Phys. Rev. D54, 1525-1534 (1996)

21. Ferrara, S., Kallosh, R., Strominger, A.: $N=2$ extremal black holes. Phys. Rev. D52, 54125416 (1995)

22. Gibbons, G.W., Kallosh, R., Kol, B.: Moduli, scalar charges, and the first law of black hole thermodynamics. Phys. Rev. Lett. 77, 4992-4995 (1996)

23. Gibbons, G.W., Maeda, K.i.: Black holes and membranes in higher dimensional theories with dilaton fields. Nucl. Phys. B298, 741 (1988)

24. Gibbons, G.W., Wiltshire, D.L.: Black holes in Kaluza-Klein theory. Ann. Phys. 167, 201 (1986)

25. Goldstein, K., Iizuka, N., Jena, R.P., Trivedi, S.P.: Non-supersymmetric attractors. Phys. Rev. D72, 124,021 (2005)

26. Horowitz, G.T., Strominger, A.: Counting states of near-extremal black holes. Phys. Rev. Lett. 77, 23682371 (1996)

27. Kallosh, R., Linde, A.D., Ortin, T., Peet, A.W., Van Proeyen, A.: Supersymmetry as a cosmic censor. Phys. Rev. D46, 5278-5302 (1992)

28. Kallosh, R., Sivanandam, N., Soroush, M.: Exact attractive non-bps stu black holes. Phys. Rev. D74, 065,008 (2006) 
29. Kallosh, R., Sivanandam, N., Soroush, M.: The non-bps black hole attractor equation. JHEP 03, 060 (2006)

30. Kaplan, D.M., Lowe, D.A., Maldacena, J.M., Strominger, A.: Microscopic entropy of $n=2$ extremal black holes. Phys. Rev. D55, 4898-4902 (1997)

31. Kaura, P., Misra, A.: On the existence of non-supersymmetric black hole attractors for two-parameter Calabi-Yau's and attractor equations. Fortsch. Phys. 54, 1109-1141 (2006)

32. Larsen, F.: Rotating Kaluza-Klein black holes. Nucl. Phys. B575, 211-230 (2000)

33. Lopes Cardoso, G., Wit, B.de, Mohaupt, T.: Corrections to macroscopic supersymmetric black-hole entropy. Phys. Lett. B451, 309-316 (1999)

34. Maldacena, J.M.: Black holes in string theory [hep-th/9607235] (1996)

35. Maldacena, J.M.: D-branes and near extremal black holes at low energies. Phys. Rev. D55, 76457650 (1997)

36. Mann, R.B., Marolf, D.: Holographic renormalization of asymptotically flat spacetimes. Class. Quant. Grav. 23, 2927-2950 (2006)

37. Mohaupt, T.: Black hole entropy, special geometry and strings. Fortsch. Phys. 49, 3-161 (2001)

38. Morse, P.M., Feshbach, H.: Methods of Theoretical Physics. Part I. McGraw-Hill, New York (1953)

39. Ortin, T.: Extremality versus supersymmetry in stringy black holes. Phys. Lett. B B422, 93-100 (1998)

40. Sabra, W.A.: General static $n=2$ black holes. Mod. Phys. Lett. A12, 2585-2590 (1997)

41. Sabra, W.A.: Black holes in $n=2$ supergravity theories and harmonic functions. Nucl. Phys. B510, 247-263 (1998)

42. Sahoo, B., Sen, A.: Btz black hole with Chern-Simons and higher derivative terms. JHEP 07, 008 (2006)

43. Sahoo, B., Sen, A.: Higher derivative corrections to non-supersymmetric extremal black holes in $n=2$ supergravity. JHEP 09, 029 (2006)

44. Sen, A.: Black hole entropy function and the attractor mechanism in higher derivative gravity. JHEP 09, 038 (2005)

45. Sen, A.: Entropy function for heterotic black holes. JHEP 03, 008 (2006)

46. Shapere, A.D., Trivedi, S., Wilczek, F.: Dual dilaton dyons. Mod. Phys. Lett. A6, 2677-2686 (1991)

47. Strominger, A.: Macroscopic entropy of $n=2$ extremal black holes. Phys. Lett. B383, 39-43 (1996)

48. Strominger, A., Vafa, C.: Microscopic origin of the Bekenstein-Hawking entropy. Phys. Lett. B379, 99-104 (1996)

49. Taylor Washington, I.: Adhering 0-branes to 6-branes and 8-branes. Nucl. Phys. B508, 122-132 (1997)

50. Tripathy, P.K., Trivedi, S.P.: Non-supersymmetric attractors in string theory. JHEP 03, 022 (2006)

51. de Wit, B., Lauwers, P.G., Van Proeyen, A.: Lagrangians of $n=2$ supergravity-matter systems. Nucl. Phys. B255, 569 (1985)

52. de Wit, B., Van Proeyen, A.: Potentials and symmetries of general gauged $n=2$ supergravity: YangMills models. Nucl. Phys. B245, 89 (1984) 